\title{
Riqueza e endemismo de espécies de Baptistonia (Orchidaceae), no Brasil
}

\author{
Guy R. Chiron ${ }^{1}$
}

Recebido: 19.06.2008; aceito: 27.08.2009

\begin{abstract}
Species richness and endemism of species of Baptistonia (Orchidaceae, Oncidiinae) in Brazil). Species endemism and species richness are complementary concepts usually used in biodiversity evaluation. They can be useful tools for efficiently defining conservation areas. In the present study, they are applied to the Brazilian Atlantic Forest, based on an orchid group endemic to this biome, the genus Baptistonia. The study of the species richness shows one very rich area (The Serra do Castelo, ES) and six rich areas. Three of these areas show an endemism index greater than 1. Eight areas, most important for biodiversity conservation, are proposed. Hierarchical relationships of endemism between nine phytogeographical areas are evaluated based on parsimony analysis of endemicity. This analysis confirms: (a) that two important endemism centers can be recognized in the southern ecological part of the Atlantic Forest, and (b) together with the phylogenetic relationships within the genus Baptistonia, that the Serra do Castelo appears to be the diversification center of the genus.
\end{abstract} Key words: Atlantic Forest, biodiversity conservation, endemicity

RESUMO - (Riqueza e endemismo de espécies de Baptistonia (Orchidaceae), no Brasil). O endemismo e a riqueza das espécies são medidas complementares da biodiversidade e atributos úteis para uma definição eficaz de áreas de preservação. Estes atributos são aqui aplicados à Floresta Atlântica brasileira, baseados em um grupo de orquídeas endêmicas deste bioma, o gênero Baptistonia. A análise da riqueza revela uma região particularmente rica (a Serra do Castelo, ES) e seis outras regiões ricas. Três destas regiões apresentam índice de endemismo superior a 1. Oito áreas prioritárias para conservação são definidas. Relações hierárquicas de endemismo entre estas áreas são avaliadas com base em análise de parcimônia de endemicidade. Do conjunto destas relações e da hipótese filogenêtica do gênero Baptistonia deduz-se que a origem deste gênero se situa na Serra do Catelo. Além do mais, esta análise confirma a existência de dois grandes centros de endemismo na zona ecológica sul da Mata Atlântica.

Palavras-chave: conservação da biodiversidade, endemismo, Mata Atlântica

\section{Introdução}

Os conceitos de endemismo e de riqueza em espécies são usualmente utilizados quando se quer avaliar a importância de uma região em termos de preservação de um bioma. Estes dois conceitos são complementares e foi sugerido que as áreas de forte endemismo e as áreas de grande riqueza de espécies não necessáriamente coincidem (Prendergast et al. 1993).A identificação dos padrões de biodiversidade apresenta conseqüências importantes para a conservação das plantas, pois a conjunção do endemismo e da riqueza em espécies aumenta a eficiência das áreas de preservação, principalmente nas florestas tropicais (Gentry 1992). Este enfoque permitiu propor vários "hotspots" para a conservação da biodiversidade em geral (Reid 1998, Myers et al. 2000) ou escolher áreas privilegiadas para a conservação deste ou daquele grupo de organismos (Kerr 1997).
A riqueza em número de espécies é uma medida fundamental da diversidade regional e subtende os modelos ecológicos e as estratégias de conservação (Magurran 1988). Este valor (riqueza em número de espécies) mede o número de espécies de um grupo biológico dado, que podem ser observadas em uma dada região. A literatura é rica em dados sobre esse conceito e sobre as dificuldades de como estima-lo (Walther \& Morand 1998, Hellmann \& Fowler 1999, Gotelli \& Colwell 2001, Ackerman et al. 2007, Smidt et al. 2007). O conceito de endemismo foi também amplamente discutido para diversos táxons em diversas áreas geográficas, tanto para quantificá-lo (Usher 1986, Kerr 1997) como para comparar uma régião à outra (Ron 2000, Bisconti et al. 2001, Linder et al. 2005, Porzecanski \& Cracraft 2005, Moreno et al. 2006). O presente estudo se baseia na avaliação destes dois conceitos, riqueza e endemismo, para o gênero Baptistonia Barb. Rodr. (Orchidaceae), como

1. Université Lyon 1, Herbiers (LY), 69622 Villeurbanne Cedex, França.g.r.chiron@wanadoo.fr 
contribuição à escolha de áreas prioritárias para sua preservação.

Baptistonia pertence à subtribo Oncidiinae e é endêmico das florestas ombrófilas densas que se encontram nas regões costeiras (Mata Atlântica), no sul e no sudeste do Brasil, e na bacia hidrográfica Paraná-Paraguai-Uruguai, que ocupa o extremo norte da Argentina, o sudeste do Paraguai e o oeste dos estados brasileiros do sul. $\mathrm{O}$ gênero foi descrito por Barbosa Rodrigues em 1877 e aplicado a todas as espécies de Oncidium Sw. seção Waluewa (Regel) Schltr. por Chiron \& Castro Neto (2004). Chiron (2007a) relacionou 21 espécies de Baptistonia, que ocupam uma área que vai do vale do rio Jacuí (RS, $30^{\circ} \mathrm{S}$, Município de São Pedro do Sul) até as colinas da Serra Geral (BA, $15^{\circ} \mathrm{S}$, Municípios de Camacan e Pau Brasil). Com base em dados morfológicos (Chiron 2007b), moleculares e químicos (Chiron et al. 2009), Chiron (2008) transferiu para o gênero Baptistonia, as espécies Oncidium venustum Drappier $(=O$. trulliferum Lindl.) e Oncidium coloratum Koeniger \& J.G. Weinmann.

\section{Material e métodos}

Base de dados - Os dados relativos às 23 espécies (ver os nomes destas assim como os nomes dos autores na tabela 1) foram obtidos em herbários, além de dados coletados em observações botânicas efetuadas pelo autor. O material testemunha foi depositado nos herbários LY e UEFS (acrônimos segundo Holmgren \& Holmgren 1998).

A primeira etapa (escolha das regiões exploradas) foi obtida por informações geográficas encontradas na literatura (Pabst \& Dungs 1977, Senghas 1997, Toscano-de-Brito, dados não publicados) e por indicaçãos fornecidas por vários contatos brasileiros. O cruzamento destas informações indica que não se encontra Baptistonia ao norte do paralelo Sul- $15^{\circ}$. No presente trabalho, as pesquisas foram ampliadas até a Serra de Jiboia (Município de Santa Teresinha, BA, ca. $12^{\circ} 30^{\prime} \mathrm{S}$ ), na extremidade da Serra Geral, para confirmar esta conclusão. Oliveira Filho \& Fontes (2000) mostraram que a Mata Atlântica compreende duas zonas florísticas distintas, respectivamente ao norte e ao sul de uma zona de transição situada ao sul da Bahia, sendo esta diferenciação provavelmente causada por variações em temperatura e regime de chuvas. O limite norte da área de distribuição do gênero Baptistonia corresponde a esta zona de transição e a ausência de Baptistonia ao norte é provavelmente devida ao mesmo motivo. Conseqüentemente, foram consultados os herbários brasileiros destas regiões: HAS, HB, ICN, MBM, PACA, R, RB, SP, UEC, UEFS, além de alguns herbários europeus $(\mathrm{G}, \mathrm{K}, \mathrm{LY}$, $\mathrm{P}, \mathrm{W}, \mathrm{WU})$.

Estes dados permitiram produzir um mapa das coletas, representando o número de amostras coletadas em uma quadrícula de $1^{\circ} \times 1^{\circ}$ e um mapa das espécies, representando o número de espécies coletadas por quadrícula. Este último mapa indica, portanto, a riqueza de espécies. O mapa de base da região geográfica concernente foi desenhado a partir de IBGE (2002) e o traçado das quadrículas sobreposto manualmente.

Índice de endemismo e análise de complementariedade - É possível atribuir a cada quadrícula um índice de endemismo que permite avaliar as diferenças de endemismo entre as diversas células. A quantificação do endemismo foi proposta por Usher (1986). Como apresentado por Kerr (1997), este método consiste em contar, para cada táxon i, o número Nì de quadrículas onde foi coletado; em seguida, para cada quadrícula $\mathrm{j}$, calcula-se um índice EIj igual à soma dos valores $1 / \mathrm{Ni}$ correspondendo aos táxons presentes nesta quadrícula:

$$
\mathrm{EIj}=\Sigma 1 / \mathrm{Ni}
$$

Este índice é útil para diferenciar quadrículas igualmente ricas em número de espécies, mas com diferentes graus de endemismo (Moreno et al. 2006). Peterson \& Watson (1998) puseram em evidência o problema de distinção entre uma definição regional do endemismo e uma definição global, porém, esse problema não interfere no presente trabalho, no qual a abrangência do estudo cobre a totalidade da área de ocorrência das espécies de Baptistonia.

A análise de complementariedade permite identificar as quadrículas que se complementam em termos de presença de espécies, isto é, que apresentam um maior número de espécies não presentes nas quadrículas já selecionadas. Assim, permite determinar as aréas mais propícias à conservação. A análise visa à preservação das espécies concernentes com um mínimo de quadrículas. Esta foi efetuada utilizando o algorítimo descrito por Rebelo \& Sigfried (1992) e Rebelo (1994), o qual consiste em escolher inicialmente a quadrícula que contém o maior número de espécies; em seguida, a que contém o maior número de espécies não presentes na primeira e assim por diante. Uma atenção especial deve ser dada às espécies amplamente distribuídas, sobretudo se são 
Tabela 1. Amostras de Baptistonia examinadas

Table 1. Baptistonia samples used in this study.

\begin{tabular}{|c|c|}
\hline \multicolumn{2}{|r|}{ Baptistonia albinoi (Schltr.) Chiron \& V.P. Castro } \\
\hline SP25185 & BRASIL. SANTA CATARINA: Joinville, II-1930, Hoehne s.n. \\
\hline SP31577 & BRASIL. SANTA CATARINA: Capivary, IV-1933, Spannagel 365 \\
\hline SP46470 & BRASIL. Rio Grande do Sul: Taquara, Gramado, III-1942, Sturmhoefel s.n. \\
\hline MBM209548 & BRASIL. PARANÁ: Três Barras, Rio Guarani, II-1993, Marques s.n. \\
\hline MBM99608 & BRASIL. PARANÁ: Morretes, Canoa Furada, alt. 100 m, II-1985, Hatschbach \& Cordeiro 48966 \\
\hline MBM6225 & BRASIL. PARANÁ: Campina Grande do Sul, Ribeirão Grande, II-1968, Hatschbach 18540 \\
\hline MBM48898 & BRASIL. PARANÁ: Campina Grande do Sul, II-1967, Hatschbach 16001 \\
\hline MBM48897 & BRASIL. PARANÁ: Piraquara, III-1953, Hatschbach 3235 \\
\hline MBM48896 & BRASIL. PARANÁ: Lapa, III-1952, Hatschbach 3190 \\
\hline MBM29392 & BRASIL. PARANÁ: S. Mateus do Sul, Fluviópolis, rio Iguaçu, III-1974, Kummrow 420 \\
\hline MBM22869 & BRASIL. PARANÁ: Quitandinha, Pangaré, III-1972, Hatschbach 29356 \\
\hline MBM2174 & BRASIL. Paraná: Campina Grande do Sul, Serra Capivari Grande, III-1967, Hatschbach 16212 \\
\hline MBM24831 & BRASIL. PARANÁ: Guarapuava, Palmerinha, XII-1972, Hatschbach 31041 \\
\hline MBM120474 & BRASIL. PARANÁ: São Mateus do Sul, II-1987, Sousa 636 \\
\hline MBM260715 & BRASIL. PARANÁ: Morretes, Mata Atlantica, II-2001, Carneiro 1054 \\
\hline HB60757 & BRASIL. SANTA CATARINA: Palhoça, Morro do Cambirela, 300m, IV-1972, Bresolin 559 \\
\hline HB2134 & BRASIL. PARANÁ: Lapa, III-1952, Hatschbach 3190 \\
\hline HB702 & BRASIL. Rio GRANDE Do Sul: pr. Lagoa dos Quadros, s.d., Dutra s.n. \\
\hline HB997 & BRASIL. Santa Catarina: Palhoça, Capivari, III-1951, Rohr 2119 \\
\hline HB1010 & BRASIL. SANTA CATARINA: Ilha S. Catarina, IV-1950, Rohr 2127 \\
\hline HB70228 & BRASIL. PARANÁ: pr. São Jeronimo da Serra, fl. cult. X-1979, Seidel 1257 \\
\hline HB11335 & BRASIL. PARANÁ: Lapa, Roseira, 910 m, III-959, Leinig 124 \\
\hline HB20325 & BRASIL. S ANTA CATARINA: Porto União, 750 m, IV-1962, Reitz \& Klein 12804 \\
\hline HB19305 & BRASIL. PARANÁ: Tibagi, fl. cult. V-1960, Feigl ex Leinig 194 \\
\hline HB41727 & BRASIL. PARANÁ: ca. 100 km SW de Guarapuava, III-1967, Lindeman \& Haas 4694 \\
\hline HB41504 & BRASIL. PARANÁ: Campina Grande do Sul, Serra Capivari, III-1967, Hatschbach 16212 \\
\hline HB56530 & BRASIL. SANTA CATARINA: estrada dos Aparados da serra a Ararangua, ca. 500 m, fl. cult. IV-1970, Brichta s.n. \\
\hline HB61131 & BRASIL. PARANÁ: pr. São Jerônimo, fl. cult. X-1974, Seidel 1114 \\
\hline HB56633 & BRASIL. Santa Catarina: São Miguel do Oeste, Canela Gaucha, 700 m, III-1964, Klein 5046 \\
\hline HB43111 & BRASIL. PARANÁ: Campina Grande do Sul, Riberão Grande, II-1968, Hatschbach 18540 \\
\hline HB60983 & BRASIL. ParanÁ: São Mateus do Sul, rio Iguaçu, III-1974, Kummrov 420 \\
\hline HB66727 & BRASIL. SANTA CATARINA: pr. Joinville, fl. cult. XII-1977, Seidel 1195 \\
\hline RB54006 & BRASIL. Santa Catarina: Nova Plutonia, III-1944, Glaumeinn 425 \\
\hline R103074 & BRASIL. SANTA CATARINA: Florianópolis, Rio Tavares, III-1952, s. col. \\
\hline R3403 & BRASIL. PARANÁ: Fernandes Pinheiro, III-1904, s. col. \\
\hline LY s.n. & BRASIL. São Paulo: Cotia, 100 km de Cotales, Castro Neto ex Chiron 03410 \\
\hline LY s.n. & BRASIL. PARANÁ: Serra do Mar, s.d., Gutfreund ex Chiron 0084 \\
\hline
\end{tabular}

Baptistonia brieniana (Rchb. f.) V.P. Castro \& Chiron

\begin{tabular}{ll}
\hline P00430186 & PARAGUAI. Santa Barbara près de Villa Rica, IV-1876, Balansa 719 (tipo de Oncidium verrucosissimum) \\
LY s.n. & PARAGUAI. haut Parana, X-1909, Fiebrig 6484 \\
W-R25704 & PARAGUAI. Maida Vale, X-XII-1980, s. col. \\
W-7911 & ARGENTINA. Misiones, Santa Ana, cult. no J. B. de Buenos Aires, I-1926, Parodi 8534 \\
G00024909 & PARAGUAI. Sierra de Amambay, pr. Esperança, s.d., Hassler 10379 \\
G00024907 & PARAGUAI. San Bernardino, s.d., Hassler 3417 \\
HAS82510 & BRASIL. RIo GrANDE Do SuL: Girua, IV-1961, Hagelund s.n. \\
HB1681 & ARGENTINA. Missiones, Garupa, VII-1931, Perez-Moreau 31/2116 \\
LY s.n. & PARAGUAI. pr. Vila Rica, s.d., Castro Neto ex Chiron 0001 \\
LY s.n. & PARAGUAI. pr. Vila Rica, XI-2004, Chiron 04604 \\
LY s.n. & PARAGUAI. pr. Vila Rica, XI-2004, Chiron 04611 \\
\hline & $\quad$ Baptistonia colorata (Koeniger \& J.G.Weinmann) Chiron \\
\hline LY s.n. & BRASIL. EsPíRITO SANTO: s.l., s.d., Castro Neto ex Chiron 0026 \\
LY s.n. & BRASIL. EsPíRITO SANTO: Serra do Castelo, s.d., col. Régent ex Chiron 06508 \\
\hline
\end{tabular}

Baptistonia cornigera (Lindl.) Chiron \& V.P.Castro

$\begin{array}{ll}\text { SP } & \text { PARAGUAI. Villa Rica, Joergensen } \\ \text { SP } & \text { BRASIL. SANTA CATARINA: Rio Tavanes, XII-1951, Rohr s.n. } \\ \text { P00437181 } & \text { PARAGUAI. Vallée de l'Ytacan, près de Valenzuela, 9-XI-1882, Balansa } 4539 \\ \text { K s.n. } & \text { PARAGUAI. SAn PEDRO: Alto Paraguay, Rio Japiracuai, XI-1955, Wooleton } \\ \text { K s.n. } & \text { ARGENTINA. Misiones: Iguazu, X-1978, Cutter \& Lonsdale 57333 }\end{array}$


K-L55/2 W-R17528 W-R14098 WU s.n. WU s.n. G00024880 G00024882 G00024882 G00024884 G00009349 G00024879 G00009350 G00024925 G00025042 G00024924 MBM226953 MBM48932 MBM48933 MBM6238 MBM8428 MBM24823 MBM46104 MBM73611 MBM251176

HAS 17757

HAS 19245

HAS82306

ICN s.n.

ICN s.n.

ICN s.n.

ICN s.n.

ICN47189

ICN110829

ICN136137

ICN35870

HB11162

HB20981

HB91052

HB733

HB2579

HB891

HB173

HB1337

HB1345

HB1893

HB2598

HB42755

HB43112

HB57464

HB13484

HB10695

HB47718

HB19497

HB19413

HB19412

HB18846

HB18137

HB766

HB2770

RB46084

RB43522

RB72848

RB46085

RB67713

RB193504

RB420165

LY s.n.

LY s.n.
BRASIL. São PaUlo: Serra do Mar prope Hyter, s.d., Martius s.n.

BRASIL. Minas Gerais: Serra de Piedade, s.d., s. col.

BRASIL. Rio DE JANEIRO: Corcovado, s.d., s. col.

BRASIL. São PAULO: Ilha Comprida, 5-10 m, 1901, Wettstein \& Schiffner

BRASIL. São PAUlo: Ilha Comprida, 5-10 m, 1901, Wettstein \& Schiffner

PARAGUAI. Igatimi, 1898/99, Hassler 5471

PARAGUAI. pr. Caaguazu, 1905, Hassler 9289

PARAGUAI. pr. Caaguazu, 1905, Hassler 9578

PARAGUAI. Ipé tui, Serra Maracayu, 1898/99, Hassler 5369

PARAGUAI. Ipé tui, Serra Maracayu, 1898/99, Hassler 5367

PARAGUAI. pr. Esperanza, Sierra de Amambay, 1907/08, Hassler 10379

PARAGUAI. Dep. ItaPuA: Delgado, 15km SE de Gral, 1978, Arbo et al. 1994

PARAGUAI. Paraguari, 266'S , 46²4'W, 1989, Zardini \& Aguayo 15812

PARAGUAI. Canindeyu, Carapa Salto, 68750/44500 UTM, 1997, Jimenez et al. 1934

PARAGUAI. Paraguari, Tebienary Mt., 350 m, Bernardi 18724

BRASIL. Paraná: Quedas do Iguaçu, Fazenda das Cobras, XII-1997, Carneiro 368

BRASIL. Paraná: Foz de Iguaçu, Parque Nacional Poço Preto, alt. 200 m, II-1963, Hatschbach 9924

BRASIL. Paraná: Campo Largo, I-1953, Hatschbach 2940

BRASIL. PARANÁ: Guaraqueçaba, Rio do Cedro, alt. 50 m, I-1968, Hatschbach 18506

BRASIL. ParanÁ: S. Jorge do Oeste, Rio Iguaçu, Salto Osório, XII-1968, Hatschbach 20539

BRASIL. PARANÁ: Medianeira, Flor da Serra, II-1971, Hatschbach 26483

BRASIL. PARANÁ: Paranagua, Morro do Inglês, II-1976, Hatschbach 38101

BRASIL. Paraná: Cerro Azul, Rio do Tigre, I-1982, Kummrow 1678

BRASIL. Paraná: Vila Alto, Rio Paraná, II-2000, Carneiro 885

BRASIL. Rio Grande Do Sul: São Jerônimo, mata ao longo do rio Jacui, XII-1982, Abruzzi 752

BRASIL. Rio Grande do Sul: São Pedro do Sul, I-1984, Corrêa 47

BRASIL. Rio Grande do Sul: Osório, Maquiné, XII-1987, Silveira 5575

BRASIL. Rio Grande do Sul: Taquara, Gravatai, I-1926, Dutra 955

BRASIL. Rio Grande do Sul: S. Leopoldo, XII-1930, Dutra 1121

BRASIL. Rio GRANDE do Sul: S. Leopoldo, s.d., Dutra 1145

BRASIL. Rio Grande Do Sul: Porto Alegre, s.d., Schuts 4319

BRASIL. Rio GRANDE do Sul: Santa Cruz do Sul, I-1980, Waechter 1517

BRASIL. Rio Grande Do Sul: Guiaba, XII-1993, Nunes 1382

BRASIL. Rio GRANDE Do Sul: Viamão, s.d., Musskopf 343

BRASIL. Rio Grande do Sul: Torres, Morro Azul, XII-1977, Waechter 674

BRASIL. SANTA CATARINA: Morro de Garrafãozinho, na encosta, 300 m, 06/1959, Welterseg 242

BRASIL. PARANÁ: pr. São Jerônimo da Serra, fl. cult. X-1964, Seidel 938

BRASIL. Paraná: São José das Pinhais, 12/1982, Seidel 1422

BRASIL. SANTA CATARINA: Ilha S. Catarina, fl. cult. XI-1950, Rohr s.n.

BRASIL. RIO DE JANEIRO: pr. Niteroi, fl. cult. X-1954, s. col.

BRASIL. Santa Catarina: Ilha S. Catarina, XII-1950, Rohr 2050

BRASIL. Rio Grande do Sul: Vale Real, fl. cult. XII-1950, Eichler s.n.

BRASIL. Rio GRANDE Do Sul: 20km ab Torres, at viam Torres-Osório, I-1952, Pabst 1337

BRASIL. Rio Grande do Sul: Vale do Maquiné inter Osório et Torres, s.d., Pabst 1345

BRASIL. Rio GRANDE Do Sul: Itapoã, I-1953, Karl s.n.

BRASIL. Rio Grande Do Sul: pr. Porto Alegre, Itapoã, fl. cult. XI-1954, Lindemayer s.n.

BRASIL. Paraná: S. Jorge do Oeste, Salto Osório, Rio Iguaçu, XII-1968, Hatschbach 20539

BRASIL. PARANÁ: Guaraqueçaba, Rio do Cedro, 50 m, 01/1968, Hatschbach 18506

BRASIL. Santa Catarina: Caiacanguçu, Morro da Ponta, 250 m, II-1971, Bresolin 162

BRASIL. PARANÁ: PN Iguaçu, II-1967, Pereira 5327

BRASIL. Paraná: Toledo, pr. Bom Princípio, ca. 450 m, I-1959, Leinig 74

BRASIL. Paraná: Foz de Iguaçu, XII-1967, Pabst 9030

BRASIL. PARANÁ: Baia de Guaratuba, 5 m, I-1961, Leinig 234

BRASIL. SANTA Catarina: Jacinto Machado, Sanga da Areia, 250 m, I-1960, Reitz \& Klein 9432

BRASIL. SANTA CATARINA: Jacinto Machado, Sanga da Areia, 250 m, 12/1959, Reitz \& Klein 9452

BRASIL. SANTA Catarina: Lauro Müller, Vargem Grande, 400 m, XII-1958, Reitz \& Klein 8096

BRASIL. Santa Catarina: Corupá, fl. cult. X-1959, Welter 260

BRASIL. SANTA Catarina: Ilha de S. Catarina, fl. cult. XI-1950, Rohr s.n.

BRASIL. RIO DE JANEIRO: Niterói, XI-1955, Mackay s.n.

BRASIL. RIO DE JANEIRO: Corcovado, s.d., Carris s.n.

BRASIL. Rio DE JANEIRO: Pedra dos Cabritos, 10/1940, serviço florestal s.n.

BRASIL. Rio Grande do Sul: estrada Gravatai-Sonhol(?), pr. Glorinha, 50 m, XI-1950, Antonio 733

BRASIL. Rio DE JANEIRO: Saccopam, X-1941, Carris s.n.

BRASIL. Rio DE JANEIRO: Morro do Jardim Botânico, fl. cult. X-1948, Carris s.n.

BRASIL. Rio DE JANEIRO: Pedra dos Cabritos, X-1941, s. col.

BRASIL. Rio DE JANEIRO: Mangaratiba, Rio das Pedras, margens do rio Grande, XI-2004, Saddi et al. 163

BRASIL. São PAULO: Maresias, X-2002, Chiron 02549

BRASIL. ParanÁ: pr. Iguaçu, V-2003, Chiron 03008 
LY s.n.

LY s.n.

LY s.n.

LY s.n.

LY s.n.

LY s.n.

LY s.n.

LY s.n.

LY s.n.

LY s.n.

LY s.n.
BRASIL. Paraná: pr. Iguaçu, V-2003, Chiron 03017

BRASIL. Paraná: pr. Iguaçu, V-2003, Chiron 03035

BRASIL. PARANÁ: pr. Iguaçu, V-2003, Chiron 03040

BRASIL. Paraná: pr. Iguaçu, V-2003, Chiron 03066

BRASIL. PARANÁ: pr. Iguaçu, V-2003, Chiron 03069

BRASIL. S̃̃o PAULO: Guarau, V-2005, Chiron 05206

BRASIL. São PaUlo: Santos, s.d., col. Vico ex Chiron 06576

BRASIL. Rio Grande do Sul: pr. Guiaba, II-2007, Chiron 07075

BRASIL. Rio Grande do Sul: pr. Guiaba, II-2007, Chiron 07076

BRASIL. S̃̃o PAULO: Sorocaba, II-2007, Chiron 07110

BRASIL. SÃo PAULO: Peruíbe, s.d., col. Vico ex Chiron 3062

Baptistonia cruciata (Rchb. f.) V.P. Castro \& Chiron

\begin{tabular}{|c|c|}
\hline & \\
\hline SP & BRASIL. Rio DE JANEIRO: Petrópolis, Meio da Serra, IV-1927, Spannagel 68 \\
\hline K s.n. & BRASIL. Rio dE JANEIRO: Canela, 900 m, II-1962, Richter s.n. \\
\hline HB60696 & BRASIL. EsPíRITo SANTO: pr. Indaiá, s.d., Ghillanyi 005/74 \\
\hline HB75764 & BRASIL. Rio dE JANEIRo: PN Tijuca, IV-1980, Toscano 57 \\
\hline HB60670 & BRASIL. EsPíRITo SANTo: Domingos Martins, IV-1974, Kautsky 430 \\
\hline HB66352 & BRASIL. EsPírito SANTo: Venda Nova, XII-1976, Zappi 8 \\
\hline HB462 & BRASIL. RIo DE JANEIRO: Petrópolis, ca. 800 m, XII-1949, Pabst 462 \\
\hline HB60695 & BRASIL. Rio de JANEIro: Teresóplis, Morro dos Penitentes, V-1974, Ghillany 004/74 \\
\hline HB1007 & BRASIL. Rio DE JANEIRO: Serra da Estrela, ad viam Rio-Petrópolis, ca. 400 m, V-1951, Pabst s.n. \\
\hline HB390 & BRASIL. EsPírITo SANTo: pr. Vitoria, s.d., "fl. 08-09”, Novais s.n. \\
\hline HB11241 & BRASIL. RIO DE JANEIRO: Teresópolis, fl. cult. XII-1958, Abendroth P125 \\
\hline HB8526 & BRASIL. São PAULO: Serra do Cantareira, 1000 m, XII-1911, Brade 6261 \\
\hline HB20590 & BRASIL. EsPíRITO SANTO: Marechal Floriano, 550 m, fl. cult. III-1964, Kautsky 15 \\
\hline HB57185 & BRASIL. Espírito SANTO: Conceção do Castello, BR 262 km 121, pr. Venda Nova, 700 m, XI-1970, Kautsky 303 \\
\hline HB43403 & BRASIL. EsPíRITO SANTO: Pedra Azul, 1000 m, II-1970, Kautsky 232 \\
\hline HB57234 & BRASIL. São PaUlo: Pinhal, X-1949, Gonçalves s.n. \\
\hline HB75761 & BRASIL. EsPíRITO SANTO: Alfredo Chaves, S. Bento de Urânia, 1000 m, III-1983, Kautsky 804 \\
\hline RB354656 & BRASIL. Rio DE JANEIRO: Nova Friburgo, Macaé de Cima, XII-1997, Miller et al. s.n. \\
\hline RB369376 & BRASIL. Rio DE JANEIRO: Nova Iguaçu, ao longo do Rio São Pedro, 700 m, fl. cult. V-2002, Moraes et al. 475 \\
\hline RB14852 & BRASIL. São PaUlo: Jaboticabal, I-1918, Frazão s.n. \\
\hline MBML8199 & BRASIL. Espírito SANTO: Santa Teresa, Est. Biológica Sta. Lúcia, VII-1995, Fraga s.n. \\
\hline MBML8200 & BRASIL. EspíRITo SANTO: Santa Teresa, Est. Biológica Sta. Lúcia, III-1995, Fraga s.n. \\
\hline MBML19335 & BRASIL. EsPíRITO SANTO: Santa Maria de Jetibá, Pedra de Garrafão, VII-2003, Bergers.n. \\
\hline LY s.n. & BRASIL. Rio DE JANEIRO: pr. Nova Friburgo, V-2005, Chiron 05411 \\
\hline LY s.n. & BRASIL. RIO DE JANEIRO: pr. Nova Friburgo, V-2005, Chiron 05441 \\
\hline LY s.n. & BRASIL. RIO DE JANEIRO: pr. Nova Friburgo, V-2005, Chiron 05446 \\
\hline LY s.n. & BRASIL. RIO DE JANEIRO: pr. Nova Friburgo, V-2005, Chiron 05451 \\
\hline LY s.n. & BRASIL. RIO DE JANEIRO: pr. Nova Friburgo, V-2005, Chiron 05452 \\
\hline LY s.n. & BRASIL. Espírito SANTO: Marechal Floriano, s.d., col. Uhl ex Chiron 06520 \\
\hline LY s.n. & BRASIL. Espírito SANTO: Marechal Floriano, s.d., col. Uhl ex Chiron 06521 \\
\hline LY s.n. & BRASIL. EsPíRITo SANTO: Marechal Floriano, s.d., col. Uhl ex Chiron 06527 \\
\hline \multicolumn{2}{|r|}{ Baptistonia damacenoi Chiron \& V.P. Castro } \\
\hline MBML6118 & BRASIL. Espírito SANTo: Santa Teresa, VIII-1990, Kollmann s.n. \\
\hline MBML8668 & BRASIL. EsPírIto SANTO: Santa Leopoldina, Rio do Norte, VIII-1998, Kollmann s.n. \\
\hline MBML12368 & BRASIL. Espírito SANTO: Santa Teresa, Est. Biológica Sta Lúcia, IV-2000, Kollmann s.n. \\
\hline MBML12796 & BRASIL. EspírITo SANTo: Cariacica, Res. de Duas Bocas, VII-2003, Fraga s.n. \\
\hline MBML21323 & BRASIL. EsPírITo SANTO: Santa Teresa, Nova Lombardia, V-2005, Fontana s.n. \\
\hline MBML24835 & BRASIL. Espírito SANTo: Santa Teresa, Rio Saltinho, VII-2005, Kollmann s.n. \\
\hline MBML28331 & BRASIL. EspíRITo SANTo: Santa Leopoldina, Morro Agudo, III-2008, Vervloet s.n. \\
\hline K s.n. & BRASIL. Espírito SANTO: s.l., IX-1894, s. col. \\
\hline LY s.n. & BRASIL. Espírito SANTO: pr. Domingos Martins, V-2003, Chiron 03208 \\
\hline LY s.n. & BRASIL. Espírito SANTo: Domingos Martins, s.d., Dominguez ex Chiron 06540 \\
\hline LY s.n. & BRASIL. EsPírITo SANTO: Venda Nova, IX-2006, col. Aloisio ex Chiron 06542 \\
\hline LY s.n. & BRASIL. EsPírITo SANTO: Venda Nova, IX-2006, col. Aloisio ex Chiron 06543 \\
\hline LY s.n. & BRASIL. EsPírito SANTO: pr. Conceição do Castello, II-2008, Chiron 08157 \\
\hline LY s.n. & BRASIL. Espírito SANTo: pr. Conceição do Castelo, s.d., Frey 746 \\
\hline LY s.n. & BRASIL. EsPíRITO SANTO: pr. Conceição do Castelo, s.d., Frey 1033 \\
\hline LY s.n. & BRASIL. EsPírITo SANTO: pr. Domingos Martins, s.d., col. Schunk ex Chiron 03399 \\
\hline LY s.n. & BRASIL. Espírito SANTO: Conceição do Castelo, II-2008, Chiron 08157 \\
\hline LY s.n. & BRASIL. EsPíRITO SANTO: Conceição do Castelo, II-2008, Chiron 08160 \\
\hline LY s.n. & BRASIL. EsPíRITO SANTO: Conceição do Castelo, II-2008, Chiron 08165 \\
\hline
\end{tabular}


Baptistonia echinata Barb. Rodr.

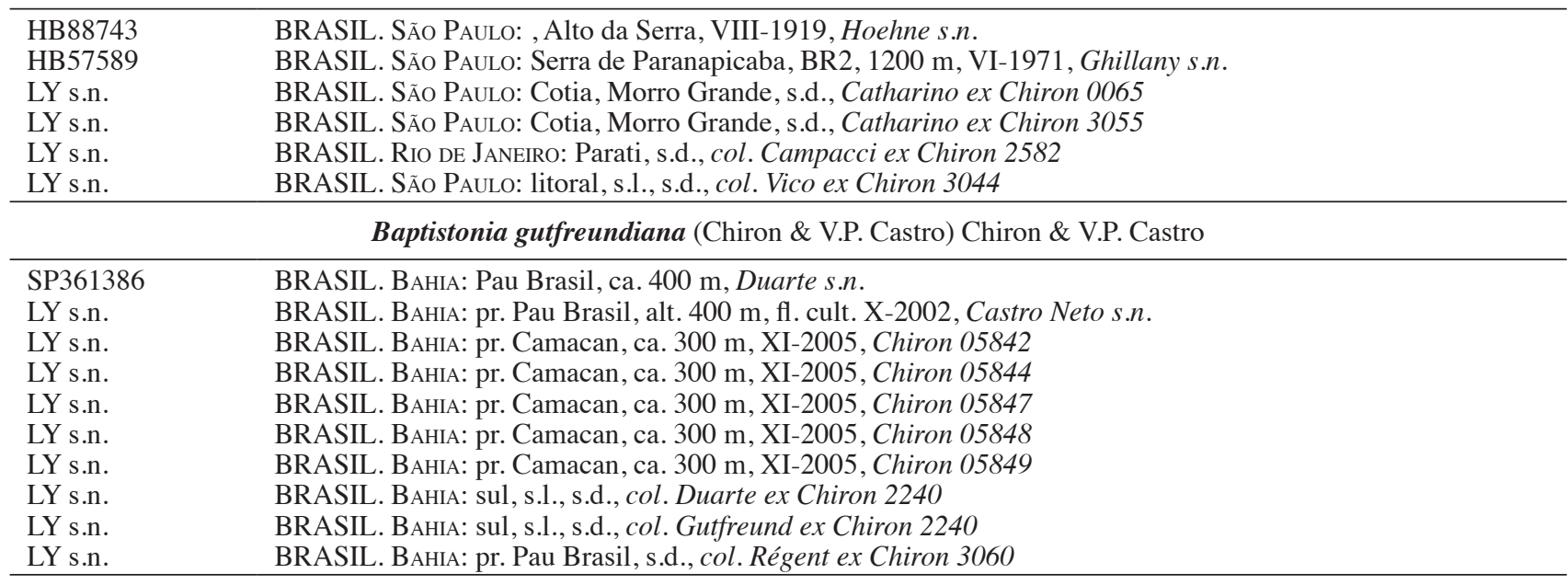

Baptistonia kautskyi (Pabst) V.P. Castro \& Chiron

\begin{tabular}{|c|c|}
\hline HB57972 & BRASIL. EsPíRITO SANTO: Vargem Alta, fl. cult. XI-1972, Seidel 1010 \\
\hline HB72862 & BRASIL. EspíRIto SAnto: São Bento de Urania, Alfredo Chaves, XI-1987, Kautsky \& Toscano 397 \\
\hline HB66729 & BRASIL. EsPíRITO SANTO: pr. Victor Hugo, fl. cult. XII-1977, Seidel 1197 \\
\hline HB66365 & BRASIL. EsPíRITO SANTO: pr. Domingos Martins, 600-800 m, XII-1976, Kautsky 560 \\
\hline HB66728 & BRASIL. EsPíRITo SANTO: pr. Victor Hugo, fl. cult. XII-1977, Seidel 1196 \\
\hline LY s.n. & BRASIL. EsPíRITO SANTO: pr. Domingos Martins, V-2003, Chiron 03194 \\
\hline LY s.n. & BRASIL. Espírito SANTo: pr. Domingos Martins, V-2003, Chiron 03329 \\
\hline LY s.n. & BRASIL. EsPírITo SANTO: Marechal Floriano, Córrego da Fortuna, XI-2004, Chiron 04813 \\
\hline LY s.n. & BRASIL. EspírITo SANTO: Marechal Floriano, Córrego da Fortuna, XI-2004, Chiron 04814 \\
\hline LY s.n. & BRASIL. EspírITo SANTO: Marechal Floriano, Córrego da Fortuna, XI-2004, Chiron 04816 \\
\hline LY s.n. & BRASIL. EspírITO SANTO: Marechal Floriano, XI-2005, Chiron 05769 \\
\hline LY s.n. & BRASIL. EsPíRITO SANTO: Conceição do Castelo, s.d., Frey 741 \\
\hline LY s.n. & BRASIL. EsPíRITo SANTo: pr. Conceição do Castelo, s.d., Frey 1079, ES \\
\hline LY s.n. & BRASIL. EsPíRITO SANTO: pr. Marechal Floriano, s.1., s.d., col. Uhl ex Chiron 0019 \\
\hline LY s.n. & BRASIL. Espírito SANTO: pr. Venda Nova do Imigrante, s.d., col. Vazzoler ex Chiron 0018 \\
\hline
\end{tabular}

Baptistonia leinigii (Pabst) V.P. Castro \& Chiron

$\begin{array}{ll}\text { SP334938 } & \text { BRASIL. PARANÁ: Ortigueira, } 800 \mathrm{~m}, \mathrm{~V}-1999, \text { Campacci MAC198 } \\ \text { HB57361 } & \text { BRASIL. PARANÁ: Ortigueira, Serra do Mulato, fl cult. III-V-1971, Leinig } \\ \text { HB57968 } & \text { BRASIL. PARANÁ: pr. Ventania, III-1972, Seidel 1027 } \\ \text { LY s.n. } & \text { BRASIL. PARANÁ: Ortigueira, Serra dos Mulatos, II-2007, Chiron } 07009 \\ \text { LY s.n. } & \text { BRASIL. PARANÁ: Ortigueira, Serra dos Mulatos, II-2007, Chiron 07010 } \\ \text { LY s.n. } & \text { BRASIL. PARANÁ: Ortigueira, Serra dos Mulatos, II-2007, Chiron 07012 } \\ \text { LY s.n. } & \text { BRASIL. PARANÁ: Ortigueira, Serra dos Mulatos, II-2007, Chiron 07014 } \\ \text { LY s.n. } & \text { BRASIL. PARANÁ: Ortigueira, Serra dos Mulatos, II-2007, Chiron 07015 } \\ \text { LY s.n. } & \text { BRASIL. PARANÁ: Ortigueira, Serra dos Mulatos, II-2007, Chiron 07017 }\end{array}$

Baptistonia lietzei (Regel) Chiron \& V.P. Castro

SP BRASIL. São Paulo: Amparo, 1926, Recch 19

SP BRASIL. RIO DE JANEIRO: Itatiaia, IV-1931, Zikan s.n.

SP47427 BRASIL. Minas GERAIs: Lavras, Heringer 931

SP37055 BRASIL. EsPíRITO SANTO: Itabapoana, XII-1936, Mazzini 8

SP5448 BRASIL. Minas Gerais: Passa Quatro, IV-1921, Zikán 3

SP4506

SP25435

SP330887

SP330891

UEC138965

P00430213

P00438805

MBM20225

MBM20225

MBM301398

MBM5043

MBM262667

HB4515

BRASIL. Minas Gerais: Poços de Caldas, XI-1920, Hoehne s.n.

BRASIL. PARANÁ: Jaguariahyra, IX-1929, Hoehne s.n.

BRASIL. Minas Gerais: Santana do Riacho, Serra do Cipó, fl. cult. XII-1989, Bicalho s.n.

BRASIL. PARANÁ: Guaira, Sete Quedas, fl. cult. X-1997, Bicalho s.n.

BRASIL. S̃̃o PAUlo: Jundiai, Serra do Japi, s.d., Singer 98/86

BRASIL. Rio DE JANEIRO: Rio Macahé, 1845, Riedel 12

BRASIL. PARANá: Paraná, Campina Grande do Sul, Riberão Grande, II-1968, Hatschbach 18540

BRASIL. PARANÁ: Sengés, Rod. S. Antonio-Itararé, 10/1971, Hatschbach 27191

BRASIL. Paraná: Maringá, Horto Florestal, X-1965, Hatschbach 12945

BRASIL. PARANÁ: Tuneiras do Oeste, XI-2004, Caxambu 583

BRASIL. PARANÁ: Cianorte, Rio Ivai, Fazenda Lagoa, 10/1966, Hatschbach 15187

BRASIL. Paraná: Fênix, Vila Rica do Espirito Santo, X-2001, Borgo 1212

BRASIL. Minas GeRAIS: Caxambu, fl. cult. X-1957, Voll s.n. 
HB11361

HB69220

HB1860

RB139064

RB193512

RB1101

RB193515

RB46564

RB375029

RB375040

RB43299

P00437349

P00437192

K s.n.

R35911

R107937

R3763

LY s.n.

LY s.n.

LY s.n.

LY s.n.

LY s.n.

LY s.n.

LY s.n.

LY s.n.

LY s.n.

LY s.n.

LY s.n.
BRASIL. Minas GERAIS: Caxambu, IX-1959, Heringer s.n.

BRASIL. EspírITo SANTo: Riberão do Meio, 12/1978, Zappi 115

BRASIL. São PaUlo: pr. Ubatuba, Serra do Mar, 12/1952, Walter 42

BRASIL. Rio DE JANEIRO: Petrópolis, ca. 900m, 04/1968, Sucre 2679 \& Braga 519

BRASIL. Rio DE JANEIRO: Petropolis, Serra da Estrela, 600 m, IV-1979, Martinelli 5778

BRASIL. RIo DE JANEIRO: Itatiaya, margens do rio Maromba, 900 m, III-1921, Campos-Porto 1037

BRASIL. Rio DE JANEIRO: Itatiaya, s.d., Brade s.n.

BRASIL. Rio DE JANEIRO: Itatiaya, 800-1000 m, III-1942, Brade 17248

BRASIL: Bahia: Vitória da Conquista, 900 m, fl. cult. X-2002, Toscano 2124

BRASIL. Rio DE JANEIRO: Sta Maria Madalena, 1000 m, fl. cult. X-2002, Cattan 164

BRASIL. Rio De JANEIRo: Serra dos Orgãos, Picada do Rancho Frio, 1300 m, fl. cult. XI-1940, Brade 16741

PARAGUAI. GuaIRA: Colonia Independencia, X-1967, Lourteig 1970

PARAGUAI. GuaIRA: Colonia Independencia, X-1967, Lourteig 1970

BRASIL. Rio DE JANEIRO: Serra dos Orgãos, VII-1878, s. col.

BRASIL. RIo DE JANEIRO: Serra do Itatiaia, IV-1926, s. col.

BRASIL. Rio DE JANEIRO: Serra dos Orgãos, 900-1000 m, V-1953, s. col.

BRASIL. Rio DE JANEIRO: Teresópolis, Serra dos Órgãos, V-1917, s. col.

BRASIL. Rio DE JANEIRO: Serra dos Orgãos, s.d., col. Binot ex Chiron 2283

BRASIL. São PAulo: Salesópolis, s.d., Chiron 3036

PARAGUAI. pr. Vila Rica, XI-2004, Chiron 04605

PARAGUAI. pr. Vila Rica, XI-2004, Chiron 04607

PARAGUAI. pr. Vila Rica, XI-2004, Chiron 04608

PARAGUAI. pr. Vila Rica, XI-2004, Chiron 04609

PARAGUAI. pr. Vila Rica, XI-2004, Chiron 04616

PARAGUAI. pr. Vila Rica, XI-2004, Chiron 04619

BRASIL. São PAULO: Presidente Prudente, s.d., col.Biorchids ex Chiron 06568

BRASIL. Paraná: Ortigueira, Serra dos Mulatos, II-2007, Chiron 07026

BRASIL. ParanÁ: Ortigueira, Serra dos Mulatos, II-2007, Chiron 07027

Baptistonia nitida (Barb. Rodr.) V.P. Castro \& Chiron

\begin{tabular}{|c|c|}
\hline $\mathrm{HB}$ & BRASIL. Rio DE JANEIRO: Serra do Barata, 800 m, s.d., Fraga ex Königer WK-98 \\
\hline HB68942 & BRASIL. RIO DE JANEIRO: Serra da Coroa Grande, fl. cult. IX-1978, Fraga s.n. \\
\hline HB63371 & BRASIL. BAHIA: margens do rio Pardo, X-1975, Silva s.n. \\
\hline HB75478 & BRASIL. EsPíRITO SANTO: Domingos Martins, fl. cult. XI-1987, Kautsky 952 \\
\hline HB1812 & BRASIL. EsPírITO SANTO: s.1., fl. cult. XI-1952, Brade ES21 \\
\hline RB64248 & BRASIL. EsPíRITO SANTO: Vargem Alta, fl. cult. X-1948, Brade 19145 \\
\hline SP & BRASIL. EsPírito SANTO: s.l., s.d., Mazzini ex Instituto de Botânica 54077 \\
\hline LY s.n. & BRASIL. Espírito SANTo: Domingos Martins, s.d., col. Ebani ex Chiron 06530 \\
\hline LY s.n. & BRASIL. Espírito SANTo: Domingos Martins, s.d., col. Ebani ex Chiron 06532 \\
\hline LY s.n. & BRASIL. Espírito SANTo: Domingos Martins, s.d., col. Schunk ex Chiron 2591 \\
\hline LY s.n. & BRASIL. Espírito SANTO: Domingos Martins, s.d., col. Schunk ex Chiron 2592 \\
\hline LY s.n. & BRASIL. Espírito SANTo: Domingos Martins, s.d., col. Schunk ex Chiron 2594 \\
\hline LY s.n. & BRASIL. Espírito SANTo: Domingos Martins, s.d., col. Schunk ex Chiron 2596 \\
\hline LY s.n. & BRASIL. Espírito SANTo: Domingos Martins, s.d., col. Dominguez ex Chiron 3 \\
\hline
\end{tabular}

Baptistonia pabstii (Campacci \& C. Espejo) V.P. Castro \& Chiron

SP334523

LY s.n.

LY s.n.
BRASIL. Rio DE JANEIRO: Casimiro de Abreu, s.d., Régent s.n.

BRASIL. Rio DE JANEIRO: pr. Macaé, IV-2006, col. Régent ex Chiron 3042

BRASIL. RiO DE JANEIRO: pr. Macaé, IV-2006, col. Régent ex Chiron 3059

Baptistonia pubes (Lindl.) Chiron \& V.P. Castro

SP27306
P00437537
ICN46295
HB79388
HB675
HB61010
HB451
HB127
HB24
HB128
HB70402
RB375034
RB136378
RB110029
RB67824
RB193511
RB141032

BRASIL. Rio de JANEIRO: Tijuca, IX-1923, col. Voll s.n.

BRASIL. RIO DE JANEIRO: Rio de Janeiro, V-1857, Regnell s.n.

BRASIL. Rio DE JANEIRO: Guanabara, VIII-1968, Sucre 3567

BRASIL. RIO DE JANEIRO: Rio de Janeiro, IX-1996, Pereira 3

BRASIL. Rio DE JANEIRO: Tijuca, IV-1950, Pabst 675

BRASIL. RIO DE JANEIRO: Guanabara, VIII-1974, Pabst 9598

BRASIL. Rio DE JANEIRO: Tijuca, X-1949, Pabst 451

BRASIL. Rio DE JANEIRO: Tijuca, IX-1950, Pabst 127

BRASIL. Rio DE JANEIRo: Serra Carioca, s.d., Pabst 24

BRASIL. Rio DE JANEIRO: Tijuca, IX-1950, Pabst 128

BRASIL. RIO DE JANEIRO: Guanabara, Mata da Lagoinha, VIII-1968, Sucre 3567

BRASIL. Rio DE J ANEIRO: Tijuca, 400 m, fl. cult. X-2002, Cattan 141

BRASIL. RIO DE JANEIRO: Guanabara, VIII-1967, Sucre 1619

BRASIL. Rio DE JANEIRO: Rio de Janeiro, Mesa do Imperador, IX-1958, Pereira et al. 4287

BRASIL. RIO DE JANEIRO: Morro Queimado, IX-1949, Brade \& Apparicio 20077

BRASIL. Rio DE JANEIRO: Tijuca (Paulo et Virginia), IX-1940, s. col.

BRASIL. Rio DE JANEIRO: Guanaraba, Mata da Lagoinha, VIII-1968, Sucre 3567 
RB140295 RB53491 RB246135 RB419212 RB183896 RB267288 R43891 K s.n. R107351

LY s.n.

LY s.n.

LY s.n.

LY s.n.

LY s.n.
BRASIL. Rio DE JANEIRO: Guanabara, Mata do Pae Ricardo, VIII-1968, Fonseca s.n. BRASIL. RIO DE JANEIRO: pico do Tijuca, fl. cult. IX-1943, Brade 17383 BRASIL. Rio DE J ANEIRO: Magé, Paraiso, VI-1986, Oliveira 105

BRASIL. RIO DE JANEIRO: Corcovado, s.d., s. col.

BRASIL. Rio DE JANEIRO: PN de Tijuca, VIII-1978, ca. 500 m, Carvalho 64

BRASIL. Rio DE JANEIRO: Rio de Janeiro, Morro do Sumaré, X-1987, Giordano et al. 346

BRASIL. RIO DE JANEIRO: Rio de Janeiro, VIII-1945, s.d., s. col.

BRASIL. Rio DE JANEIRO: Angra dos Reis, I. de Portobello, s.d., s. col.

BRASIL. RIO DE JANEIRO: Magé, s.d., s. col.

BRASIL. Rio DE JANEIRO: pr. Macaé, IV-2006, col. Régent ex Chiron 06506

BRASIL. Rio DE JANEIRO: s.l., s.d., col. Vico ex Chiron 3034

BRASIL. RIO DE JANEIRO: pr. Macaé, s.1., IV-2006, col. Régent ex Chiron 3038

BRASIL. Rio DE JANEIRO: pr. Macaé, s.1., IV-2006, col. Régent ex Chiron 3046

BRASIL. Rio DE J JNEIRO: pr. Macaé, s.l., IV-2006, col. Régent ex Chiron 3053

\begin{tabular}{|c|c|}
\hline HB23044 & BRASIL. RIO DE JANEIRO: Teresopolis, Serra dos Orgãos, IV-1962, Santos 1193, Fromm 1175 \& Trinta 151 \\
\hline HB40621 & BRASIL. RIO DE JANEIRO: Petrópolis, fl. cult. V-1965, Verboonen s.n. \\
\hline R s.n. & BRASIL. RIO DE JANEIRO: Teresópolis, IV-1962, Santo S1193 \\
\hline LY s.n. & BRASIL. RIo DE JANEIRO: Nova Friburgo, ca. 1000 m, V-2005, Chiron 05415 \\
\hline LY s.n. & BRASIL. Rio DE JANEIRO: Nova Friburgo, Boa Esperança, ca. 1000 m, V-2005, Chiron 05466 \\
\hline LY s.n. & BRASIL. RIo DE JANEIRO: Nova Friburgo, RJ, ca. 1000 m, V-2005, Chiron 05487 \\
\hline LY s.n. & BRASIL. RIO DE JANEIRO: Nova Friburgo, RJ, ca. 1000 m, V-2005, Chiron 05498 \\
\hline
\end{tabular}

SP363181

HAS 82509

HAS 82312

HAS82511

PACA84970

PACA50678

ICN s.n.

ICN s.n.

HB621

HB701

RB323279

LY s.n.

LY s.n.

LY s.n.

LY s.n.

LY s.n.

LY s.n.
BRASIL. Rio Grande do Sul: Novo Hamburgo, Vale dos Sinos, 1990, Cassola s.n.

BRASIL. Rio Grande do Sul: Torres, Serra, alt. 500 m, I-1975, Hagelund 9044

BRASIL. Rio Grande Do Sul: Veranópolis, Serra, XII-1985, Silveira 3045

BRASIL. Rio Grande do Sul: Tenente Portela, III-1988, Silveira 8793

BRASIL. Rio Grande do Sul: A Montenegro, S. Salvador, alt. 600 m, IV-1963, Sehnem 82010

BRASIL. Rio Grande do Sul: S. Salvador, III-1950, A. Sehnem 4895

BRASIL. Rio Grande do Sul: Maquiné, s.d., Dutra 925

BRASIL. Rio Grande Do Sul: S. Sebastião do Cai, II-1928, Dutra 1102

BRASIL. Rio Grande do Sul: pr. Gramado, Valle flumen Cahy, III-1952, Pabst 621

BRASIL. Rio Grande do Sul: pr. Torres, s.d., Dutra s.n.

BRASIL. Rio GRANDE Do SUL: Santa Maria, Cerrito, III-1980, Ferreira et al. 1099

BRASIL. Rio Grande do Sul: Faroupilha, Forromecco, ca. 300 m, II-2007, Chiron 07060

BRASIL. Rio Grande do Sul: Faroupilha, Forromecco, ca. 300 m, II-2007, Chiron 07061

BRASIL. Rio Grande do Sul: Faroupilha, Forromecco, ca. 300 m, II-2007, Chiron 07062

BRASIL. Rio Grande do Sul: Faroupilha, Forromecco, ca. 300 m, II-2007, Chiron 07063

BRASIL. Rio Grande do Sul: Faroupilha, Forromecco, ca. 300 m, II-2007, Chiron 07069

BRASIL. Rio Grande do Sul: Faroupilha, Forromecco, ca. 300m, II-2007, Chiron 07070

\section{Baptistonia sarcodes (Lindl.) Chiron \& V.P. Castro}

SP361423

P00437595

P00437594

K s.n.

K s.n.

K s.n.

W-R12051

WU s.n.

WU s.n.

MBM10148

MBM218812

MBM48899

MBM85474

HB1742

HB18132

HB63040

HB009

RB431296

RB289527

UEFS s.n.

LY s.n.

LY s.n.

LY s.n.

LY s.n.
BRASIL. São PAulo: Guarujá, V-1999, Pinheino 168

BRASIL. Paraná: Paraná, Jaguariahyva, 740 m, (1910?), Dusén 10852

BRASIL. ParanÁ: Paraná, Jaguariahyva, 740 m, Dusén 15960

BRASIL. Paraná: Paraná, Jaguariahyua, 1910, Dusén 10852

BRASIL. Paraná: Paraná, Jaguariahyua, 1910, Dusén 10859

BRASIL. PARANá: Paraná, Jaguariahyua, 740 m, 1914, Dusén 15960

BRASIL. SÃo PAULO: Bertioga, 2-25 m, VI-1901, Wettstein \& Schiffner s.n.

BRASIL. São PAUlo: Bertioga, 5-25 m, 1901, Wettstein \& Schiffner s.n.

BRASIL. São PaUlo: Bertioga, 5-25 m, 1901, Wettstein \& Schiffner s.n.

BRASIL. PARANÁ: Ortigueiras, Serra dos Mulatos, XI-1968, Hatschbach 20344

BRASIL. Paraná: Ribeirão do Pinhal, XI-1996, Carneiro 261

BRASIL. PARANÁ: Tibagi, s.d., Hatschbach 2804

BRASIL. PARANÁ: Guaratuba, Rio Itararé, XI-1983, Kummrow 2379

BRASIL. São PAULO: pr. Pindamonhangaba, X-1952, Welter s.n.

BRASIL. PARANÁ: Curiuva, fl. cult. XI-1959, Leinig 154

BRASIL. SÃo PAULO: litoral norte, fl. cult. XI-1974, Waras s.n.

BRASIL. Rio de JaneIro: Paulo de Frontin, Granja Rodeio, s.d., Pabst 009

BRASIL. RIO DE JANEIRO: Paracambi, X-2006, Rezende s.n.

BRASIL. Rio De JANEIRO: Parati, 250 m, XI-1990, Giordano 888, Toscano 611 et al.

BRASIL. São PAULO: Bertioga, Guaratuba, XI-2004, Chiron 04850

BRASIL. São PAULO: Bertioga, Guaratuba, XI-2004, Chiron 04852

BRASIL. S̃̃o PAULO: Bertioga, Guaratuba, XI-2004, Chiron 04853

BRASIL. São PaUlo: Peruibe, Guarau, V-2005, Chiron 05218

BRASIL. São PAULO: Itapira, Serra de Itapira, s.d., col. Biorchids ex Chiron 06570 
LY s.n.

LY s.n.

LY s.n.

UEFS s.n.
BRASIL. Paraná: Ortigueira, Serra dos Mulatos, II-2007, Chiron 07094

BRASIL. Minas Gerais: Camanducaia, s.d., Jardim Botânico de São Paulo A492

BRASIL. São Paulo: Peruibe, Jureia, s.d., Jardim Botânico de São Paulo 13785

BRASIL. Paraná: Ortigueira, Serra dos Mulatos, II-2007, Chiron 07025

\begin{tabular}{|c|c|}
\hline \multicolumn{2}{|r|}{ Baptistonia silvana (V.P. Castro \& Campacci) V.P. Castro \& Chiron } \\
\hline $\begin{array}{l}\text { SP341854 } \\
\text { LY s.n. } \\
\text { LY s.n. } \\
\text { LY s.n. } \\
\text { LY s.n. } \\
\text { LY s.n. } \\
\text { UEFS s.n. }\end{array}$ & $\begin{array}{l}\text { BRASIL. BAHIA: Una, Serra das Trempes, s.d., Silva s.n. } \\
\text { BRASIL. BAHIA: Buerarema, s.d., Chiron 0057, Edmundo s.n. } \\
\text { BRASIL. B AHIA: São José de Vitória, Serra Boa, XI-2005, Chiron } 05794 \\
\text { BRASIL. BAHIA: São José de Vitória, Serra Boa, XI-2005, Chiron } 05795 \\
\text { BRASIL. BAHIA: São José de Vitória, Serra Boa, XI-2005, Chiron } 05800 \\
\text { BRASIL. BAHIA: Buerarema, s.d., col. Régent ex Chiron 06509 } \\
\text { BRASIL. BAHIA: São José de Vitória, Serra Boa, XI-2005, Chiron } 05807\end{array}$ \\
\hline \multicolumn{2}{|r|}{ Baptistonia truncata (Pabst) Chiron \& V.P. Castro } \\
\hline $\begin{array}{l}\text { SP50366 } \\
\text { UEC140294 } \\
\text { HB19517 } \\
\text { HB57268 } \\
\text { RB46563 } \\
\text { RB193516 } \\
\text { LY s.n. } \\
\text { LY s.n. } \\
\text { LY s.n. } \\
\text { LY s.n. } \\
\text { LY s.n. } \\
\text { LY s.n. } \\
\text { LY s.n. }\end{array}$ & 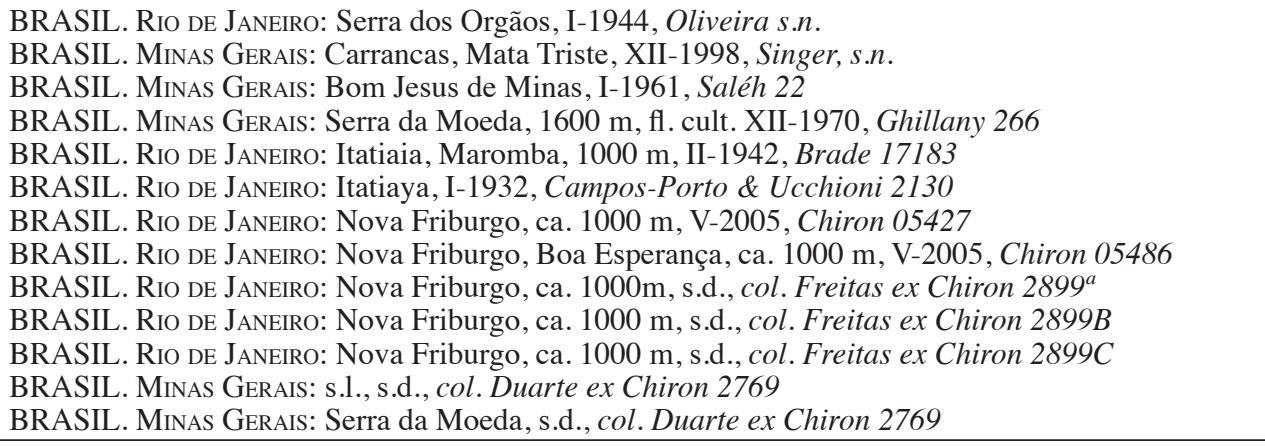 \\
\hline \multicolumn{2}{|r|}{ Baptistonia uhlii Chiron \& V.P. Castro } \\
\hline $\begin{array}{l}\text { LY s.n. } \\
\text { LY s.n. } \\
\text { LY s.n. }\end{array}$ & $\begin{array}{l}\text { BRASIL. EspírITo SANTO: Domingos Martins, Pedra do Tamanco, XI-2004, col. Uhl ex Chiron } 2689 \\
\text { BRASIL. EspírITo SANTO: Domingos Martins, Pedra do Tamanco, s.d., col. Uhl ex Chiron } 06513 \\
\text { BRASIL. EsPírITo SANTO: Domingos Martins, Pedra do Tamanco, s.d., col. Uhl ex Chiron } 06534\end{array}$ \\
\hline \multicolumn{2}{|r|}{ Baptistonia velteniana V.P. Castro \& Chiron } \\
\hline $\begin{array}{l}\text { LY s.n. } \\
\text { LY s.n. } \\
\text { LY s.n. }\end{array}$ & $\begin{array}{l}\text { BRASIL. Espí́ito SANTO: Domingos Martins, ca. } 500-700 \text { m, s.d., Kautsky s.n. } \\
\text { BRASIL. EspírITO SANTO: Domingos Martins, s.d., col. Uhl ex Chiron 0028 } \\
\text { BRASIL. Espírito SANTO: Domingos Martins, s.d., col. Uhl ex Chiron 0029 }\end{array}$ \\
\hline \multicolumn{2}{|r|}{ Baptistonia venusta (Drappier) Chiron } \\
\hline MBM48902 & BRASIL. PARANÁ: Guaratuba, IV-1953, Hatschbach 3272 \\
\hline MBM10717 & BRASIL. PARANÁ: Paranagua, Pico Turbo, III-1969, Hatschbach 21257 \\
\hline MBM48903 & BRASIL. PARANÁ: Morretes, Rio Mãe Catira, IV-1957, Hatschbach 3928 \\
\hline MBM7757 & BRASIL. ParanÁ: Antonina, Cab. Rio Faisqueira, alt. 50 m, III-1968, Hatschbach 18703 \\
\hline MBM48901 & BRASIL. PARANÁ: Guaratuba, Pedra Branca de Araraquara, XI-1961, Hatschbach 8781 \\
\hline MBM7758 & BRASIL. PARANÁ: Guaraqueçaba, Rio do Cedro, alt. 50-100 m, I-1968, Hatschbach 18153 \\
\hline ICN44114 & BRASIL. Rio Grande do Sul: Torres, XI-1979, Waechter 1192 \\
\hline ICN s.n. & BRASIL. Rio Grande do Sul: Maquiné, s.d., Dutra 1104 \\
\hline ICN11918 & BRASIL. Rio GRANDE DO SUL: Torres, II-1927, Dutra 918 \\
\hline HB8513 & BRASIL. São PAUlo: Iguape, Morro das Pedras, XII-/1919, Brade 8039 \\
\hline HB43393 & BRASIL. PARANÁ: Paranagua, Pico Torto, III-1969, Hatschbach 21257 \\
\hline НB4336 & BRASIL. Paraná: Morretes, Rio Mãe Catira, IV-1957, Hatschbach \& Smith 3928 \\
\hline HB3161 & BRASIL. RIO DE JANEIRO: pr. Nova Friburgo, III-1957, Vale s.n. \\
\hline HB2647 & BRASIL. SANTA CATARINA: Tijucas, Morro do Cambirela, III-1955, Rohr 2268 \\
\hline HB1984 & BRASIL. SANTA CATARINA: Ilha de S. Catarina, IV-1953, Rohr s.n. \\
\hline RB97425 & BRASIL. Rio DE JANEIRO: Barreira, Serra dos Orgãos, IV-1956, Pereira 1953 \\
\hline LY s.n. & BRASIL. São PaUlo: Peruibe, Jureia, V-2005, Chiron 05249 \\
\hline LY s.n. & BRASIL. S̃̃o Paulo: I. Cardoso, s.d., Jardim Botânico de São Paulo 11008 \\
\hline LY s.n. & BRASIL. Minas Gerais: Mariana, s.d., Jardim Botânico de São Paulo 7028 \\
\hline LY s.n. & BRASIL. Rio DE JANEIRO: Parati, s.d., Jardim Botânico de São Paulo 3273 \\
\hline LY s.n. & BRASIL. S̃̃o PAUlo: I. Cardoso, s.d., Jardim Botânico de São Paulo 12463 \\
\hline LY s.n. & BRASIL. Rio GRANDE DO Sul: s.1., s.d., col. Teobaldo ex Chiron 0108 \\
\hline LY s.n. & BRASIL. Rio GRANDE DO Sul: s.l., s.d., col. Teobaldo ex Chiron 0109 \\
\hline
\end{tabular}




\begin{tabular}{|c|c|}
\hline \multicolumn{2}{|r|}{ Baptistonia widgrenii (Lindl.) V.P. Castro \& Chiron } \\
\hline SP19514 & BRASIL. MinAS GERAIS: Ouro Fino, Canelleiras, V-1927, Hoehne s.n. \\
\hline SP46502 & BRASIL. MinAS GERAIS: Lavras, III-1942, Heringer 971 \\
\hline SP75861 & BRASIL. São PAULO: Bragança Paulista, V-1955, Handro 482 \\
\hline P s.n. & BRASIL. Minas GeraIs: Caldas, IX-1842, Regnell 1690 \\
\hline K-L 50 & BRASIL. MinAS GERAIS: s.l., s.d., Widgren 774 \\
\hline HB2982 & $\begin{array}{l}\text { BRASIL. Minas Gerais: Nova Lima, pr. Lagoa Grande, Serra da Motuca, } 1600 \text { m, II-1945, Williams \& Assis } \\
5791\end{array}$ \\
\hline HB019 & BRASIL. Minas GERAIS: S. Lourenço, II-1944, Pabst 019 \\
\hline HB57240 & BRASIL. São PAULO: Bragança Paulista, V-1955, Handro 482 \\
\hline RB62466 & BRASIL. Minas GERAIS: Caxambu, IV-1948, Dantas 6 \\
\hline RB74157 & BRASIL. São PAULO: Serra do Bocaina, 1700 m, V-1957, Brade 20925 \\
\hline RB95503 & $\begin{array}{l}\text { BRASIL. Minas GeraIs: Nova Lima, pr. Lagoa Grande, Serra da Motuca(Mutuca), } 1600 \text { m, II-1945, Williams } \\
\text { \& Assis } 5791\end{array}$ \\
\hline RB95499 & $\begin{array}{l}\text { BRASIL. Minas GeraIs: Nova Lima, pr. Lagoa Grande, Serra da Motuca(Mutuca), } 1500 \text { m, III-1945, Williams } \\
\text { \& Assis } 6204\end{array}$ \\
\hline RB193518 & BRASIL. MinAS GERAIS: sul, s.1., III-1935, Carvalho s.n. \\
\hline R35912 & BRASIL. MinAS GERAIS: Passa Quatro, IV-1929, s. col. \\
\hline LY s.n. & BRASIL. Minas GERAIS: Itajubá, s.d., col. Castro Neto ex Chiron 03407 \\
\hline LY s.n. & BRASIL. Minas GERAIS: Itajubá, s.d., col. Castro Neto ex Chiron 03408 \\
\hline LY s.n. & BRASIL. Minas GeraIs: Itajubá, s.d., col. Castro Neto ex Chiron 03409 \\
\hline
\end{tabular}

compostas de várias populações separadas entre si: a diversidade destas populações pode ser tão importante de ser conservada quanto somente a diversidade das espécies.

Análise de parcimônia por endemismo - Para avaliar as relações de endemismo entre as diferentes regiões e para identificar as regiões com alto grau de endemismo, foi utilizada a análise de parcimônia por endemismo $(\mathrm{PAE}=$ parsimony analysis of endemicity). A análise PAE, proposta por Rosen (1988), é um método da biogeografia histórica, análogo à cladistica, que procura precisar as afinidades biogeográficas da Unidades Geográficas Operacionais (Operating Geographic Units, OGUs). Muitas vezes, as OGUs são quadrículas (Morrone 1994). O mesmo foi utilizado em várias espécies animais e vegetais (Posadas \& Miranda-Esquivel 1999, Trejjo-Torres \& Ackerman 2001, Garcia-Barros et al. 2002, Morrone \& Escalante 2002, Rovito et al. 2004). Craw (1988) sugeriu utilizar áreas de endemismo predeterminadas e Silva \& Oren (1996) escolheram áreas interfluviais. No presente trabalho foram definidos como conjuntos de quadrículas adjacentes contendo geralmente as mesmas espécies e correspondendo a entidades geográficas determinadas, obtidas a partir das áreas previamente detectadas por análise de complementariedade.

Os dados de entrada da PAE consistem em uma matriz composta de OGUs e táxons, semelhante às matrizes táxons/caracteres utilizadas em filogenia na qual a ausência de um táxon em uma OGU é anotada como 0 e a sua presença como 1. Uma área hipotética na qual todos os táxons estão ausentes pode ser acrescentada como grupo externo (Rosen \& Smith 1988). Lambshead \& Paterson (1986) de fato estabeleceram que, se as espécies são consideradas como caracteres a partir dos quais se pode inferir cladogramas, então a presença de uma espécie deve ser considerada como um caráter derivado e sua ausência como um caráter ancestral.

A análise de parcimônia foi efetuada segundo o algorítimo de Fitch (1971), com auxílio do software PHYLIP 3.5 (Felsenstein 1989, 1993). A robustez do cladograma proposto foi determinada pelo teste de "bootstrap" com 1000 replicações (Felsenstein 1985). Considera-se geralmente fraco, o suporte "bootstrap", entre $50 \%$ e $70 \%$, médio entre $71 \%$ e $85 \%$ e forte se superior a $85 \%$ (Kress et al. 2002).

\section{Resultados}

Base de dados - Das 439 amostras examinadas nos herbários e identificadas, muitas não possuíam indicação precisa do local de coleta. Aquelas que possuíam, somadas às 141 plantas observadas pelo autor, permitiram considerar 436 amostras (tabela 1).

Duas espécies apresentaram ampla distribuição, doze, área de ocorrência reduzida, enquanto as restantes apresentaram área de dimensões intermediárias (tabela 2). 
Tabela 2. Superfícies de distribução das espécies de Baptistonia.

Table 2. Distribution area of the Baptistonia species.

\begin{tabular}{|l|l|}
\hline \multicolumn{1}{|c|}{ Espécies } & \multicolumn{1}{c|}{ Superficies de distribução } \\
\hline B. cornigera & cerca de $1.500 \mathrm{~km} \times$ largura da floresta \\
B. lietzei & $200.000-270.000 \mathrm{~km}^{2}$ \\
\hline B. albinoi & \\
B. brieniana & \\
B. cruciata & \\
B. echinata & \\
B. nitida & \\
B. sarcodes & \\
B. truncata & \\
B. venusta & \\
B. widgrenii & \\
\hline B. colorata & \\
B. damacenoi & \\
B. gutfreundiana & \\
B. kautskyi & \\
B. leinigii & \\
B. pabstii & menos de $10.000 \mathrm{~km}^{2}$ \\
B. pubes & \\
B. pulchella & \\
B. riograndensis & \\
B. silvana & \\
B. uhlii & \\
B. velteniana & \\
\hline & \\
\hline
\end{tabular}

Considerando a diferença de abundância natural das espécies nas áreas selecionadas, observou-se que algumas apresentaram um número reduzido de amostras: duas para B. colorata, três para B.pabstii, $B$. uhlii e $B$. velteniana, seis para $B$. echinata. A maioria das espécies é representada por 10-25 amostras, enquanto que outras de maior distribuição contêm mais de 30 amostras (até 84 para B. cornigera). Podese notar que nenhuma das espécies dos herbários europeus provém de localidades situadas na zona ecológica norte da Mata Atlântica.

A figura 1A representa o mapa de distribuição dessas amostras e pode ser sobreposta ao mapa de distribuição da floresta ombróphila densa proposto por SOSMA \& INPE (2008): as quadrículas nas quais as espécies de Baptistonia foram coletadas em grande número (mais de cinco amostras) correspondem a zonas onde as florestas são mais conservadas de acordo com este mapa.

A figura 1B fornece o número de espécies registradas em cada quadrícula. A região com a maior riqueza em espécies é constituída pelas quadrículas 10 e 11, que corespondem à Serra do Castelo (ES), com os municipios de Domingos Martins, Marechal Floriano, Alfredo Chavez, Vargem Alta, Venda Nova do Imigrante e Conceição do Castelo. Nela encontramse sete espécies: Baptistonia colorata, B. cruciata, $B$. damacenoi, B. kautskyi, B. nitida, B. uhlii e $B$. velteniana.

Nas Serras ao norte e a oeste da cidade de São Paulo, a quadrícula 26 contém cinco especies: $B$. cornigera, B. lietzei, B. cruciata, B. echinata, $B$. sarcodes. Seguem-se várias regiões com quatro espécies.

Ao sul do estado do Rio de Janeiro, três áreas geográficas diferentes podem ser identificadas: as florestas de baixa altitude (quadrícula 21) com $B$. cornigera, B. pabstii, B. pubes e B. sarcodes, as florestas da Serra do Mar (quadrícula 20) com $B$. cruciata, B. lietzei, B. pulchella e B. truncata e as Serras de Bocaina e da Mantiqueira (quadrícula 19, RJ-SP-MG), com B. lietzei, B. truncata, B. widgrenii, $B$. echinata, como B. cornigera nas baixas altitudes (abaixo de $400 \mathrm{~m}$ ).

No Estado do Paraná, na Serra dos Mulatos, a quadrícula 34 contém $B$. lietzei, $B$. sarcodes, $B$. leinigii e $B$. albinoi enquanto que, na Serra do Mar, a quadrícula 46 contém $B$. cornigera, $B$. albinoi, $B$. sarcodes e B. venusta.

Mais ao sul, na Serra Geral (RS), quadrícula 63 , emcontram-se B. albinoi, B. cornigera, $B$. riograndensis e $B$. venusta.

Índice de endemismo e análise de complementariedade - A figura 1C ilustra os resultados obtidos no cálculo dos índices de endemismo. Três áreas apresentam um índice particularmente alto, a Serra do Castelo, previamente mencionada, mas também a Serra dos Orgãos (RJ) e a Serra das Trempes (Una, BA); vêm em seguida três outras quadrículas, uma delas corresponde aos arredores da cidade do Rio de Janeiro e à parte ocidental da Serra dos Orgãos, as outras, à Serra dos Mulatos (Mun. Ortigueira, PR) e aos arredores da cidade de Villa Rica (Paraguai).

Os resultados da análise de complementariedade estão apresentados na tabela 3. Oito áreas permitem cobrir a totalidade das espécies. Duas áreas contêm a metade delas: a Serra do Castelo (ES), com sete espécies das quais cinco são endêmicas e a Serra des Orgãos (RJ), com cinco espécies das quais duas endêmicas. Aárea ${ }^{\circ} 7$ é a unica região que não contém nem uma espécie endêmica, mas encontram-se aí duas espécies não presentes nas regiões 1 a $6, B$. echinata $\mathrm{e}$ B. widgrenii. A última espécie, Baptistonia brieniana, está presente sobretudo no Paraguai, notadamente nas 

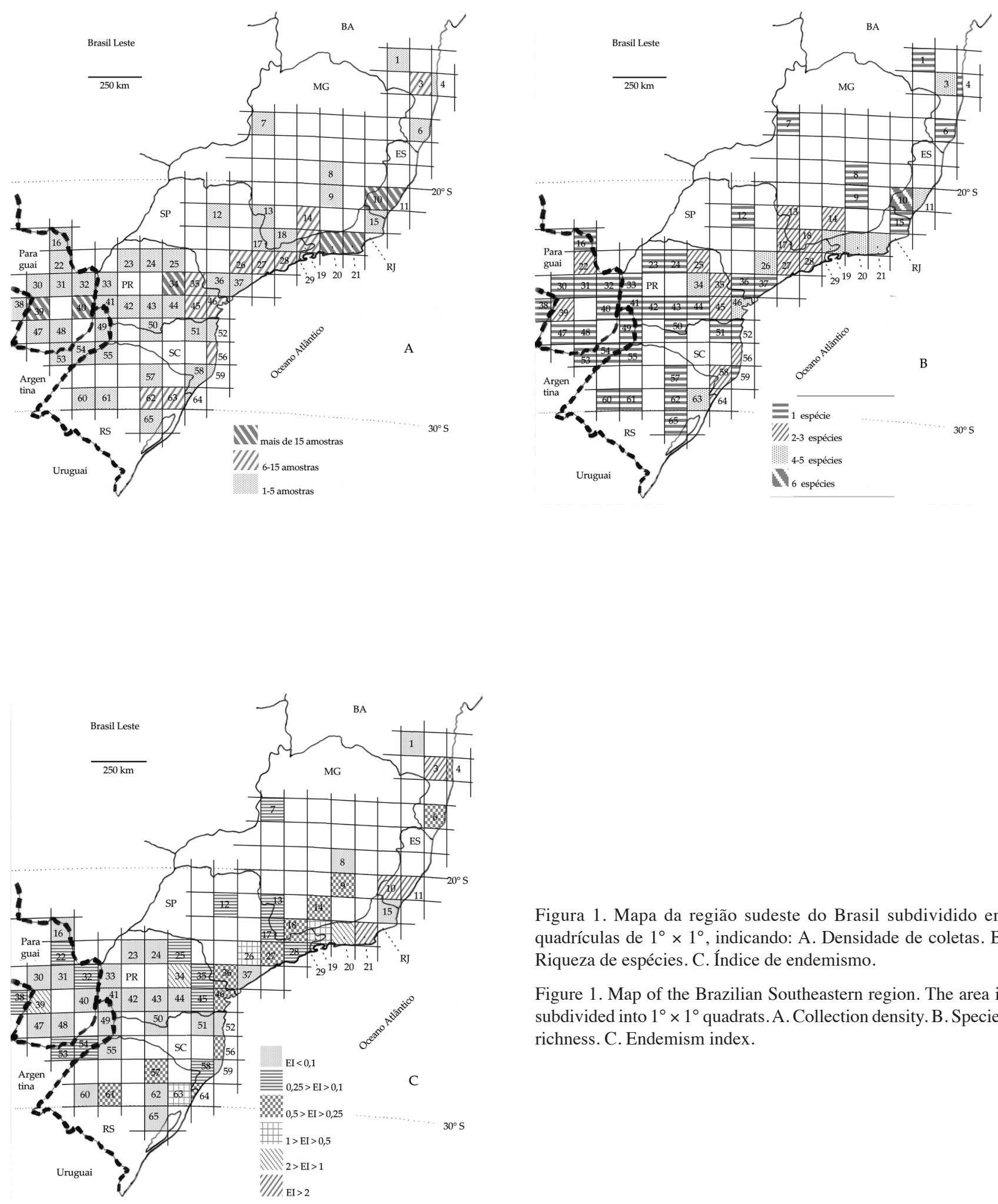

Figura 1. Mapa da região sudeste do Brasil subdividido em quadrículas de $1^{\circ} \times 1^{\circ}$, indicando: A. Densidade de coletas. B. Riqueza de espécies. C. Índice de endemismo.

Figure 1. Map of the Brazilian Southeastern region. The area is subdivided into $1^{\circ} \times 1^{\circ}$ quadrats. A. Collection density. B. Species richness. C. Endemism index. 
florestas perto de Villa Rica (Departamento Guairá) onde igualmente foi coletada uma subespécie de $B$. lietzei endêmica desta região, B. lietzei ssp. guairensis. Baptistonia brieniana foi coletada no Brasil também, na quadrícula 55 (área $\mathrm{n}^{\circ}$ 8). Tres áreas, embora ricas em espécies, não contêm nem uma espécie endêmica tampouco uma espécie não presente nas outras áreas: a Serra do Mar no Estado de São Paulo (Serra de Paranapiacaba, Serra de Cantareira e serras mais interiores: área $n^{\circ}$ 9), a Serra do Mar no Estado do Paraná (área $n^{\circ} 10$ ) e a Serra do Mar e a Serra Geral em Santa Catarina (área $n^{\circ} 11$ ).

Análise de parcimônia por endemismo - A área de distribuição do gênero Baptistonia foi dividida em nove regiões fitogeográficas (ou OGUs), baseadas nas áreas obtidas na análise de complementariedade. Estas OGUs são, do norte ao sul: 1- o sul do estado da Bahia, centralizado na Serra das Trempes (área n 3), 2- a Serra do Castelo (ES - área n ${ }^{\circ}$ 1), 3- a Serra do Mar no estado do Rio de Janeiro e ao extremo este do estado de São Paulo (a Serra dos Orgãos e a Serra do Bocaina, incluindo as áreas $n^{\circ} 2,5$ e parte da 7), 4- a Serra do Mar no estado de São Paulo (área no 9), 5- a Serra da Mantiqueira (parte da área ${ }^{\circ} 7$ ), 6- a Serra Geral no Paraná (incluindo a Serra dos Mulatos, área $n^{\circ} 4$ ), 7- a Serra do Mar no Paraná (área n 10), 8- a extremidade sul da Serra Geral (sul de Santa Catarina e norte do Rio Grande do Sul, incluindo as áreas ${ }^{\circ} 6$ e $\left.{ }^{\circ} 11\right)$ e 9- a bacia Paraná-Paraguai-Uruguai (P.P.U. - área $\left.{ }^{\circ} 8\right)$.

A tabela 4 mostra a matriz obtida a partir da presença das espécies de Baptistonia nestas regiões. Entre os 24 táxons (23 espécies mais uma subespécie), somente nove são informativos, os outros sendo endêmicos de uma única região. Nenhuma região apresenta grande desbalanço de presença: o menor número de táxons em uma região é três, o maior é oito e a média cinco. A análise de parcimônia forneceu 42 árvores mais parcimoniosas (AMPs), das quais o comprimento é de 29 . O índice de consistência IC $=0,83$, o índice de retenção IR $=0,72$; o suporte de "bootstrap" é geralmente moderado (79\%) a forte (88\%-92\%), raramente fraco $(56 \%)$. As diferenças topológicas entre as árvores são fracas. A figura 2 mostra o AMP com maior suporte de "bootstrap". Dois conjuntos de regiões com forte relação fitogeográfica são visíveis (e o sendo na totalidade dos $42 \mathrm{AMPs}$ ): o primeiro (com suporte de 88\%) formado pelas zonas florestais do estado do Espírito Santo e do sul do estado da Bahia; o segundo (com suporte de 89\%), pelo conjunto das outras zonas.

\section{Discussão}

Representatividade do material estudado - Os métodos utilizados neste estudo são instrumentos úteis para determinar áreas prioritárias para conservação, mas eles supõem que o material sobre o qual estes se baseiam, não deixa grandes áreas geográficas inexploradas. Constatou-se que o número de espécimes relacionados depende muito da espécie considerada e da quadrícula estudada. A recente descoberta de alguns táxons é uma razão do pequeno número de coletas e da falta de conhecimento: é o caso de $B$. colorata (descrita em 1994), B. pabstii (1998), B. uhlii (2006) e $B$. velteniana (2005) com duas amostras, além do material tipo. Mas também são taxons cuja área de distribuição é pequena.

As quadrículas onde se observa maior número de coletas (figura 1A) são, muitas vezes, regiões de fácil acesso (ao redor da cidade do Rio de Janeiro, onde $B$. pubes é comum ou a região de Iguaçu, na fronteira com a Argentina-Brasil-Paraguai, com B. cornigera). Outras vezes, elas correspondem a regiões estudadas pelo autor, tais como a Serra do Castelo (ES), a Serra dos Orgãos (RJ), a Serra dos Mulatos (PR), as redondezas da cidade de Villa Rica (Paraguai), onde foi efetuada grande quantidade de coletas. Mas as quadrículas, onde o número de espécimes coletados é pequeno, correspondem, na verdade, a regiões pouco propícias à presença de Baptistonia, por serem regiões mais secas e desprovidas de florestas ombrófilas densas. Ao longo do litoral estas áreas situam-se ao norte do Espírito Santo, vale do Rio Doce, ao norte do Rio de Janeiro, vale do Paraíba do Sul, e ao sul do estado de São Paulo, vale do Rio Ribeira de Iguape. Quanto à ausência de observações ao norte da Serra das Trempes (BA), pode ser explicada pelas diferenças florísticas apresentadadas pelas áreas ecológicas norte e sul da Mata Atlântica.

A boa correlação entre as densidades de espécies coletadas e as densidades de florestas residuais é uma indicação positiva da boa representatividade do material. Mas isto é válido em grande escala. Em nível mais local, visto que foram levadas em conta amostras históricas para determinar as áreas de maior valor em termos de biodiversidade, conviria assegurar-se de que as florestas nestas areas não foram destruídas pela forte progressão das atividades antrópicas. De fato, dados recentes avaliam em aproximadamente $7 \%$ os remanecentes de Mata Atlântica, mais precisamente, entre 5\% e 12\% para Tonhasca (2005) e 7,5\% para Myers (2000). Um exemplo deste tipo de problema 
Tabela 3. Análise de complementaridade: regiões fitogeográficas e espécies associadas. Espécie «nova» da região ${ }^{\circ} \mathrm{j}$ : espécie não presente nas regiões $n^{\circ} 1$ a j-1 (espécies abaixo das linhas pontilhadas).

Table 3. Complementarity analysis: phytogeographical areas and associated species. "New species" for the area $\mathrm{n}^{\circ} \mathrm{j}$ : species not recorded in the $\operatorname{areas} n^{\circ} 1$ to $n^{\circ} \mathrm{j}-1$ (placed below the dotted lines).

\begin{tabular}{|c|c|c|c|c|c|c|c|}
\hline área & $\begin{array}{c}\text { quadrículas } \\
\text { incluidas }\end{array}$ & $\begin{array}{c}\text { região } \\
\text { fitogeográfica }\end{array}$ & $\begin{array}{l}\text { número de } \\
\text { espécies }\end{array}$ & espécies & $\begin{array}{c}\mathrm{n}^{\circ} \text { de } \\
\text { espécies } \\
\text { «novas» }\end{array}$ & $\begin{array}{c}\mathrm{n}^{\circ} \text { de espécies } \\
\text { endêmicas }\end{array}$ & $\begin{array}{c}\mathrm{n}^{\circ} \text { de espécies } \\
\text { em conjunto }\end{array}$ \\
\hline 1 & 10,11 & $\begin{array}{c}\text { Serra do Castelo } \\
\text { (ES) }\end{array}$ & 7 & $\begin{array}{c}\text { B. colorata } \\
\text { B. cruciata } \\
\text { B. damacenoi } \\
\text { B. kautskyi } \\
\text { B. nitida } \\
\text { B. uhlii } \\
\text { B. velteniana }\end{array}$ & 7 & 5 & 7 \\
\hline 2 & 20,21 & $\begin{array}{c}\text { Serra dos Órgãos } \\
(\mathrm{RJ})\end{array}$ & 5 & $\begin{array}{c}\text { B. cruciata } \\
\text { ….............. } \\
\text { B. lietzei } \\
\text { B.pabstii } \\
\text { B. pulchella } \\
\text { B. truncata }\end{array}$ & 4 & 2 & 11 \\
\hline 3 & 3 & $\begin{array}{c}\text { Serra das Trempes } \\
\text { (BA) }\end{array}$ & 3 & $\begin{array}{c}\text { B. nitida } \\
\text { B.................. } \\
\text { B. gutfreundiana } \\
\text { B. silvana }\end{array}$ & 2 & 2 & 13 \\
\hline 4 & 34 & $\begin{array}{l}\text { Serra dos Mulatos } \\
\text { (PR) }\end{array}$ & 4 & $\begin{array}{c}\text { B. lietzei } \\
\text {................... } \\
\text { B. albinoi } \\
\text { B. leinigii } \\
\text { B. sarcodes }\end{array}$ & 3 & 1 & 16 \\
\hline 5 & 20 parte & Rio de Janeiro (RJ) & 3 & $\begin{array}{l}\text { B. sarcodes } \\
\text { …................ } \\
\text { B. cornigera } \\
\text { B. pubes }\end{array}$ & 2 & 1 & 18 \\
\hline 6 & 62,63 & Vale do Cai (RS) & 3 & $\begin{array}{c}\text { B. cornigera } \\
\text { …................ } \\
\text { B. riograndensis } \\
\text { B. venusta }\end{array}$ & 2 & 1 & 20 \\
\hline 7 & 18,19 & $\begin{array}{c}\text { Serras de } \\
\text { Bocaina (RJ) e da } \\
\text { Mantiqueira (RJ, } \\
\text { SP, MG) }\end{array}$ & 5 & $\begin{array}{c}\text { B. lietzei } \\
\text { B. sarcodes } \\
\text { B.truncata } \\
\text {.................... } \\
\text { B. echinata } \\
\text { B. widgrenii }\end{array}$ & 2 & 0 & 22 \\
\hline 8 & 39 & $\begin{array}{r}\text { Villa Rica } \\
\text { (Paraguai) }\end{array}$ & $\begin{array}{c}2+ \\
1 \mathrm{ssp}\end{array}$ & $\begin{array}{c}\text { B. cornigera } \\
\text {.................. } \\
\text { B. lietzei } \mathrm{ssp} . \\
\text { guairensis } \\
\text { B. brieniana }\end{array}$ & 1 & 1 & $23+1 \mathrm{ssp}$ \\
\hline
\end{tabular}




\begin{tabular}{|c|c|c|c|c|c|c|c|}
\hline área & $\begin{array}{l}\text { quadrículas } \\
\text { incluidas }\end{array}$ & $\begin{array}{c}\text { região } \\
\text { fitogeográfica }\end{array}$ & $\begin{array}{l}\text { número de } \\
\text { espécies }\end{array}$ & espécies & $\begin{array}{c}\mathrm{n}^{\circ} \mathrm{de} \\
\text { espécies } \\
\text { «novas» }\end{array}$ & $\begin{array}{c}\mathrm{n}^{\circ} \text { de espécies } \\
\text { endêmicas }\end{array}$ & $\begin{array}{c}\mathrm{n}^{\mathrm{o}} \text { de espécies } \\
\text { em conjunto }\end{array}$ \\
\hline 9 & 26 & Serra do Mar (SP) & 5 & $\begin{array}{c}\text { B. cornigera } \\
\text { B. cruciata } \\
\text { B. echinata } \\
\text { B. lietzei } \\
\text { B. sarcodes }\end{array}$ & 0 & 0 & \\
\hline 10 & 46 & Serra do Mar (PR) & 5 & $\begin{array}{c}\text { B. albinoi } \\
\text { B. cornigera } \\
\text { B. lietzei } \\
\text { B. sarcodes } \\
\text { B. venusta }\end{array}$ & 0 & 0 & \\
\hline 11 & 56,58 & Serras de SC & 3 & $\begin{array}{c}\text { B. albinoi } \\
\text { B. cornigera } \\
\text { B. venusta }\end{array}$ & 0 & 0 & \\
\hline
\end{tabular}

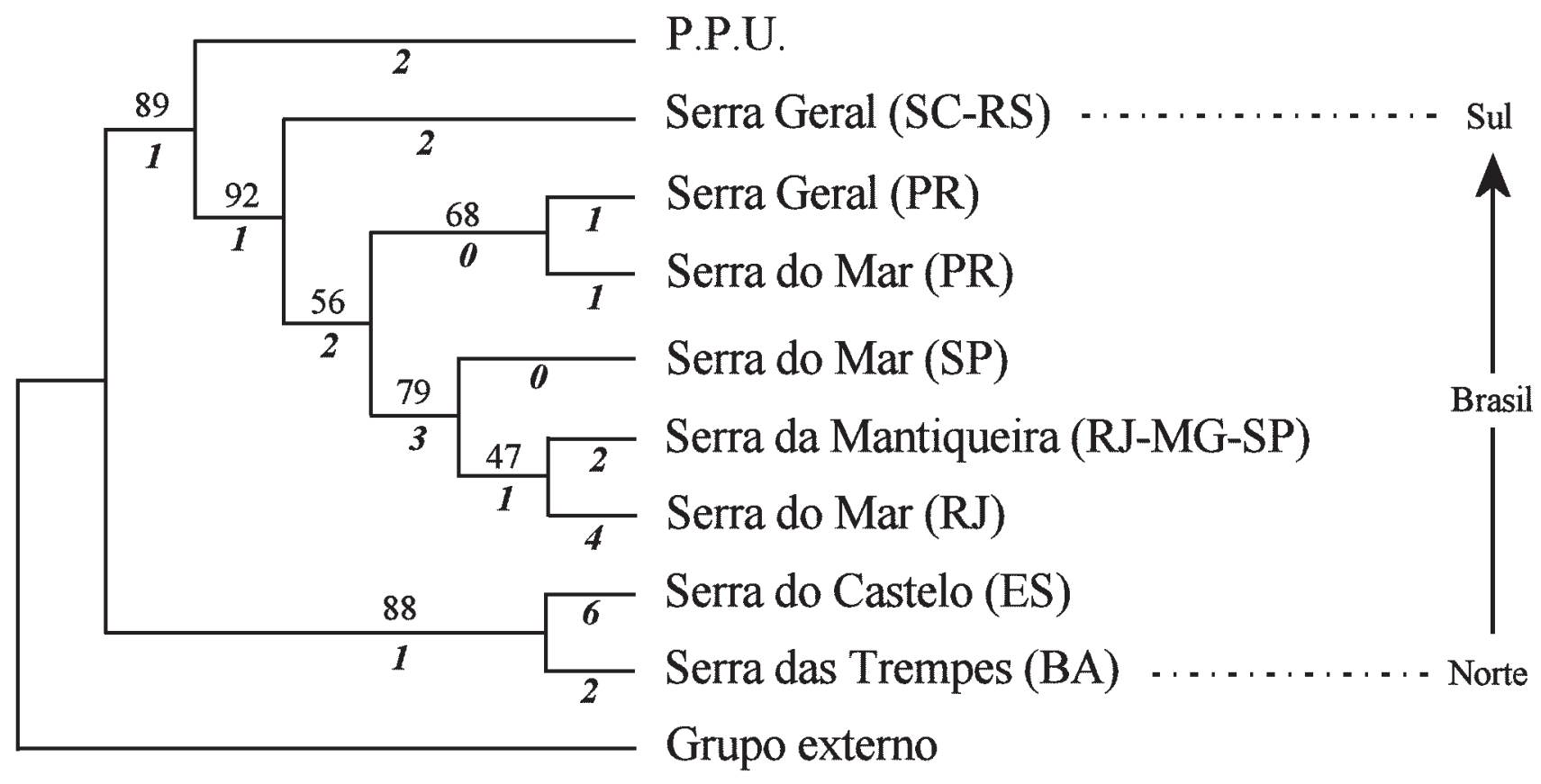

Figura 2. Árvore de maior suporte resultante da análise de parcimônia de endemicidade das regiões fitogeográficas da Mata Atlântica do Brasil, obtida a partir de espécies de Baptistonia. Os números acima dos ramos correspondem aos valores de "bootstrap", aqueles colocados abaixo dos ramos são os comprimentos dos ramos. Comprimento: 29, IC = 0,83, IR = 0,72. 42 AMPs.

Figure 2. Maximum support tree obtained from the parsimony analysis of endemicity of the phytogeographical areas of the Brazilian Atlantic Forest, based on Baptistonia species. Numbers above branches are bootstrap percentages, and those below branches are branch lengths. Length: 29, IC =0.83, IR =0.72. Number of most parsimonious trees (MPTs): 42 . 


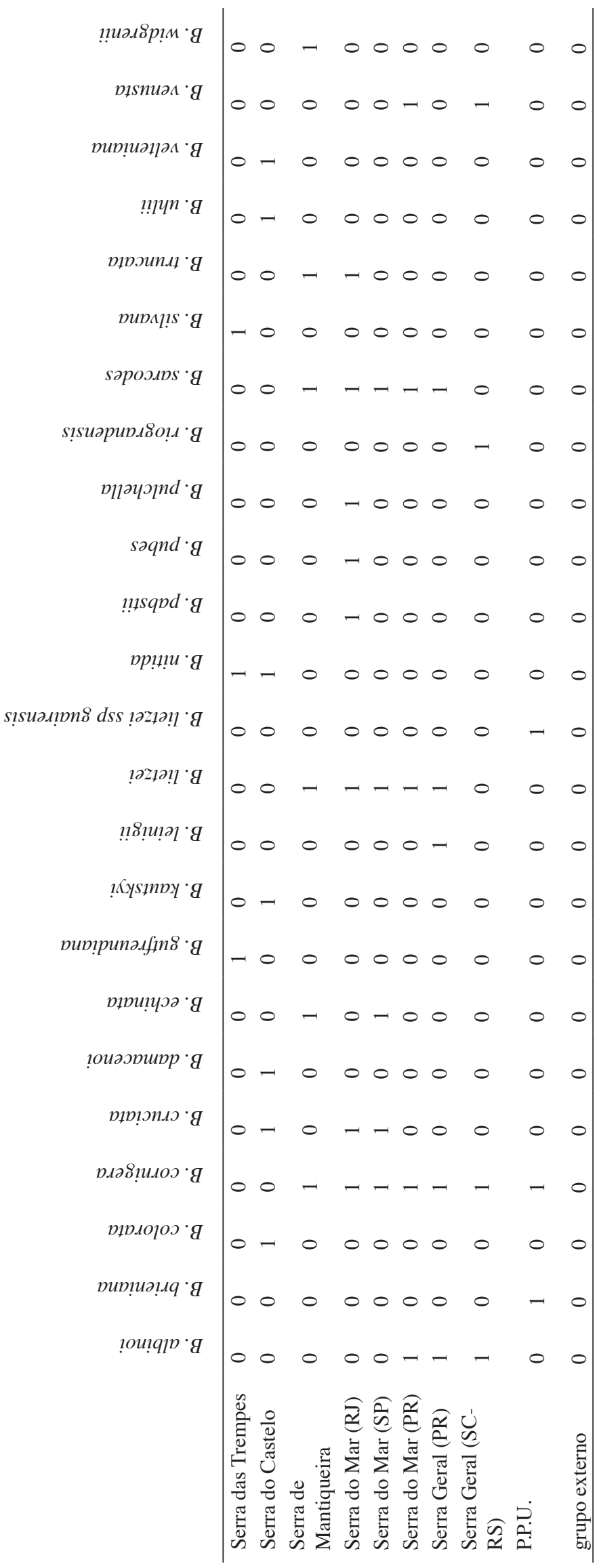


pode ser encontrado nas florestas da Serra do Castelo (ES). As superfícies de florestas primárias reduziram-se bastante pela atividade agrícola, ao ponto de o mapa de vegetação publicado em 1995 pelo Instituto Brasileiro de Geografia e Estatística (IBGE) não indicar mais nenhuma cobertura vegetal natural. Os hábitats das espécies de Baptistonia nessa região, classificada como prioridade 1 nas análises de complementariedade, são hoje muito reduzidos, o que representa um risco na conservação destas espécies, devido à redução do tamanho populational a niveis críticos.

Áreas prioritárias para a preservação - Os índices de endemismo (figura 1C) e a análise de complementariedade (tabela 3) revelaram que três regiões são particularmente importantes para a preservação da diversidade do gênero Baptistonia: a Serra do Castelo, a Serra dos Orgãos e a Serra das Trempes. Estas regiões fazem parte dos dois centros de endemismo geralmente reconhecidos (Tonhasca 2005) na área ecológica sul da Mata Atlântica. O estudo do gênero Baptistonia confirma, portanto, os resultados já publicados, os quais conferem às diversas áreas ainda cobertas por florestas, o status de zonas altamente prioritárias do ponto de vista concervação (SOSMA \& INPE 2008). Um destes centros de endemismo estende-se do sul da Bahia ao Espírito Santo e inclui a Serra das Trempes (região n ${ }^{\circ} 3$ ) e a Serra do Castelo (região $n^{\circ} 1$ ), o outro centro compreende o sul do Rio de Janeiro (regiões $n^{\circ} 2,5$ e 7).

Estes centros de endemismo estão relativamente próximos do Oceano Atlântico. As duas outras regiões importantes do Brasil, em termos de conservação de Baptistonia, são regiões interiores (região ${ }^{\circ} 4$ na Serra dos Mulatos) ou bem ao sul (região ${ }^{\circ} 6$, vale do Rio Cai) ainda não identificadas como regiões prioritárias para preservação (IBGE 2002).

Análise de parcimônia por endemismo - Os comentários que podem ser feitos sobre esta análise são de dois tipos. Primeiramente, ela ilustra duas grandes regiões na área ecológica sul da Mata Atlântica (figura 2), caracterizadas por um endemismo diferente. Uma corresponde ao centro endêmico norte desta região ecolológica (Tonhasca 2005) situado ao norte da linha de demarcação constituída pelo vale do Rio Paraíba do Sul, compreendendo o conjunto Serra das Trempes- Serra do Castelo. A proximidade florística destes dois massiços está bem sustentada pelo teste de "boostrap" (88\%).

Ao sul desta linha estão reunidas, com um bom suporte, as outras OGUs tomadas na análise. No interior deste segundo grupo de OGUs, as afinidades florísticas delimitam um subgrupo que corresponde ao centro endêmico sul da Mata Atlântica (Serra da Mantiqueira, Serra dos Orgãos, Serra da Bocaina) com extenção até a Serra de Paranapiacaba. Com um suporte de "bootstrap" de 79\%, as afinidades florísticas desta região são nitidamente mais pronunciadas que as daquelas entre as serras do Paraná (68\%).

A análise filogenética (Chiron et al.2009) baseada em caracteres morfológicos e moleculares, mostrou que Baptistonia colorata é irmã dos autros membros do gênero. Inclusive, Castro Neto \& Lacerda (2006) descreveram um gênero monoespecífico, Carriella, para abrigar essa espécie. A presença de B. colorata, espécie irmã do restante do gênero, no bloco norte da PAE é um indício de que o gênero possa ter se originado nessa área, mas mais estudos são necessários para testar essa hipótese.

\section{Agradecimentos}

O autor agradece aos curadores dos herbários visitados durante o decorrer de nossos estudos das Baptistonia : G, HAS, HB, ICN, K, LY, MBM, P, PACA, R, RB, SP, UEC, UEFS, W, WU. Agradece também os assessores anônimos que propuseram muitas melhorias.

\section{Literatura citada}

Ackerman, J.D., Trejo-Torres, J.C. \& Crespo-Chuy, Y. 2007 Orchids of the West Indies: predictability of diversity and endemism. Journal of Biogeography 34 : 779-786.

Bisconti M., Landini, W., Bianucci, G., Cantalamessa, G., Carnevale, G., Ragaini, L. \& Valleri, G. 2001. Biogeographic relationships of the Galapagos terrestrial biota: Parsimony Analyses of Endemicity based on resptiles, land birds and Scalesia land plants. Journal of Biogeography 28: 495-510.

Castro Neto, V.P. \& Lacerda, K.G. 2006. Carriella colorata. In: V.P. Castro Neto (ed.). Icones Orchidacearum Brasilienses II. V.P. Castro Neto, São Bernardo do Campo, tab. 123.

Chiron, G. 2007a. Note sur la distribution géographique de Baptistonia Barbosa Rodrigues (Orchidaceae, Oncidiinae). Richardiana 7: 145-173.

Chiron, G. 2007b. Phylogenetic analyses of the genus Baptistonia (Orchidaceae, Oncidiinae) sensu lato based on morphological characters. Journal of the Botanical Research Institute of Texas 1: 913-931.

Chiron, G. 2008. Baptistonia Barbosa Rodrigues (Orchidaceae: Oncidiinae) - Notes taxinomiques. 2. Richardiana 8: 111-127. 
Chiron, G. \& Castro Neto, V.P. 2004. Contribution à la connaissance des orchidées du Brésil. III- Rétablissement du genre Baptistonia Barbosa Rodrigues. Richardiana 4: 109-120.

Chiron, G., Oliveira, R.P., Santos, T.M., Bellvert, F., Bertrand, C. \& van den Berg, C. 2009. Phylogeny and evolution of Baptistonia (Orchidaceae, Oncidiinae) based on molecular analyses, morphology and floral oil evidences. Plant Systematics and Evolution 281: 35-49.

Craw, R. 1988, Continuing the synthesis between panbiogeography, phylogenetic systematics and geology as illustrated by empirical studies on the biogeography of New Zealand and the Chatham islands. Systematic Zoology 37: 291-310.

Felsenstein, J. 1985. Confidence limits on phylogenies: an approach using the bootstrap. Evolution 39: 783-791.

Felsenstein, J. 1989. PHYLIP - Phylogeny Inference Package (version 3.2). Cladistics, 5: 164-166.

Felsenstein, J. 1993. PHYLIP (Phylogeny Inference Package) version 3.5c. Distribuido pelo autor, University of Washington, Department of Genetics, Seatle.

Fitch W.M. 1971. Towards defining the course of evolution: Minimum change for a specific tree topology. Systematic Zoology 20: 406-416.

Garcia-Barros, E., Gurrea, P., Lucianez, M.J., Cano, J.M., Munguira, M.L., Moreno, J.C., Sainz, H., Sanz, M.J. \& Simon, J.C. 2002. Parsimony analysis of endemicity and its application to animal and plant geographical distributions in the Ibero-Balearic region (Western Mediterranean). Journal of Biogeography 29: 109-124.

Gentry, A.H. 1992. Tropical Forest Biodiversity: Distributional patterns and their conservational significance. Oikos 63: 19-28.

Gotelli, N.J. \& Colwell, R.K. 2001. Quantifying biodiversity: procedures and pitfalls in the measurement and comparison of species richness. Ecology Letters 4: 379-391.

Hellmann, J.J. \& Fowler, G.W. 1999. Bias, precision and accuracy of four measures of species richness. Ecological Applications 993: 824-834.

Holmgren, P.K. \& Holmgren, N.H. 1998 [continuously updated]. Index Herbariorum: A global directory of public herbaria and associated staff. New York Botanical Garden's Virtual Herbarium. http://sweetgum.nybg.org/ih/.

IBGE. 2002. Atlas geográfico escolar. Instituto Brasileiro de Geografia e Estatística, Rio de Janeiro.

Kerr, J.T. 1997. Species richness, endemism, and the choice of areas for conservation. Conservation Biology 11: 1094-1100.

Kress, W.J., Prince L.M. \& Williams, K.J. 2002. The phylogeny and a new classification of the gingers (Zingiberaceae): evidence from molecular data. American Journal of Botany 89: 1682-1696.
Lambshead, P.J.D. \& Paterson, G.L.G. 1986. Ecological cladistic. An investigation of numerical cladistics as a method for analising ecological data. Journal of Natural History 20: 895-909.

Linder, H.P., Kurzweil, H., Johnson, S.D. 2005. The Southern African orchid flora: composition, sources and endemism. Journal of Biogeography 32: 29-47.

Magurran, A.E. 1988. Ecological diversity and its measurement. Princeton University Press, Princeton.

Moreno, R.A., Hernandez, C.E., Rivadeneira, M.M., Vidal, M.A. \& Rozbaczylo, N. 2006. Patterns of endemism in south-eastern Pacific benthic polychaetes of the Chilean coast. Journal of Biogeography 33: 750-759.

Morrone, J.J. 1994. On identification of areas of endemism. Systematic Biology 43: 438-441.

Morrone, J.J. \& Escalante, T. 2002. Parsimony Analysis of Endemicity (PAE) of Mexican terrestrial mammals at different area units: when size matters. Journal of Biogeography 29: 1095-1104.

Myers, N., Mittermeier, R.A., Mittermeier, C.G., Fonseca, G.A.B. \& Kent, J. 2000. Biodiversity hotspots for conservation priorities. Nature 403: 853858.

Oliveira Filho, A.T. \& Fontes, M.A.L. 2000. Patterns of floristic differentiation among Atlantic forests in Southeastern Brazil and the influence of climate. Biotropica 32: 793-810.

Pabst, G.F.J.\& Dungs, F. 1977. Orchidaceae Brasilienses. II. Kurt Schmersow, Hildesheim.

Peterson, A.T. \& Watson, D.M. 1998. Problem with areal definitions of endemism: the effects of spatial scaling. Diversity and Distributions 4: 189-194.

Porzecanski,A.L. \& Cracraft, J. 2005. Cladistic analysis of distribution and endemism (CADE): using raw distribution of birds to un ravel the biogeography of the South American aridlands. Journal of Biogeography 32: 261-275.

Posadas, P. \& Miranda-Esquivel, D.R. 1999. El PAE (Parsimony Analysis of Endemicity) como una herramienta en la evaluacion de la biodiversidad. Revista Chilena de Historia Natural 72: 539-546.

Prendergast, J.R., Quinn, R.M., Lawton, J.H., Eversham,B.C.\& Gibbons, D.W. 1993. Rare species, the coincidence of diversity hotspots and conservation strategies. Nature 365: 335-337.

Rebelo,A.G. 1994. Iterative selection procedures: Centers of endemism and optimal placement of reserves. Strelitzia 1: 231-257.

Rebelo, A.G.\& Sigfried, W.R. 1992. Where should nature reserves be located in the Cape Floristic Region, South Africa? Models for the spatial configuration of a reserve network aimed at maximising the protection of diversity. Conservation Biology 6: 243-252. 
Reid, W.V. 1998. Biodiversity hotspots. Trends in ecology and evolution 13: 275-280.

Ron, S. 2000. Biogeographic area relationships of lowland Neotropical rainforest based on raw distributions of vertebrate groups. Biological Journal of the Linnean Society 71: 379-402.

Rosen, B.R. 1988. From fossils to earth history: applied historical biogeography, pp.437-481. In: A.A. Myers \& P.S. Gillers (eds.), Analytical Biogeography., Chapman \& Hall, London.

Rosen, B.R. \& Smith, A. B. 1988. Tectonics from fossils? Analysis of reef-coral and sea-urchin distributions from Late Cretaceous to Recent, using a new method. In: M.G. Audley-Charles \& A. Hallam (eds.), Gondwana and Tethys. Geological Society. Special Publication $n^{\circ}$ 37, Oxford University Press, Oxford, pp. 275-306.

Rovito, S., Arroyo, M.T.K. \& Pliscoff, P. 2004. Distributional modelling and Parsimony Analysis of Endemicity of Senecio in the Mediterranean-type climate area of Central Chile. Journal of Biogeography 31: 1623-1636.

Senghas, K. 1997. Subtribus Oncidiinae. In: Sonderabdruck aus Schlechter, Die Orchideen, 3 Auflage - 76. Paul Parey, Berlin.
Silva, J.M.C. \& Oren, D.C. 1996. Application of parsimony analysis of endemicity in Amazonian biogeography: an example with primates. Biological Journal of the Linnean Society 59: 427-437.

Smidt, E.C., Silva-Pereira, V., Borba, E.L. \& van den Berg, C. 2007. Richness, distribution and important areas to preserve Bulbophyllum in the Neotropics. Lankesterana 7: 107-113.

SOSMA \& INPE. 2008. Atlas dos remanescentes florestais da Mata Atlântica - periodo 2000-2005. Fundação SOS Mata Atlântica e Instituto Nacional de Pesquisas Espaciais, São Paulo.

Tonhasca, A. 2005. Ecologia e história natural da Mata Atlântica. Interciência, Rio de Janeiro.

Trejjo-Torres, J.C.\& Ackerman, J.D. 2001. Biogeography of the Antilles based on a parsimony analysis of orchid distributions. Journal of Biogeography 28: 775-794.

Usher, M.B. 1986. Wildlife conservation evaluation. Chapman \& Hall, London.

Walther, B.A. \& Morand, S., 1998. Comparative performance of species richness estimation methods. Parasitology 116: 395-405. 